JOURNAL OF THE AMERICAN MATHEMATICAL SOCIETY

Volume 14, Number 1, Pages 79-107

S 0894-0347(00)00351-9

Article electronically published on September 18, 2000

\title{
BOUNDEDNESS OF AUTOMORPHIC $L$-FUNCTIONS IN VERTICAL STRIPS
}

\author{
STEPHEN GELBART AND FREYDOON SHAHIDI
}

\section{INTRODUCTION}

The purpose of this paper is to prove the boundedness in vertical strips of finite width for all the $L$-functions that appear in constant terms of Eisenstein series, under a certain natural assumption on local normalized intertwining operators. As a corollary, we prove the boundedness for a number of important $L$-functions, among them the symmetric cube and triple product $L$-functions attached to cusp forms on $G L(2)$, as well as several Rankin-Selberg product $L$-functions, where the local assumption was already proved to be valid in each case. Moreover, our main theorem plays a fundamental role in establishing certain new and striking cases of functoriality ( $\mathrm{CKPSS}], \mathrm{K3}$, [KSh4). Under the same assumption, the finiteness of poles on all of $\mathbb{C}$ for all of these $L$-functions is also proved, a necessary step in the proof of boundedness in vertical strips.

More precisely, let $\mathbf{G}$ be a quasisplit connected reductive group over a number field $F$ whose ring of adeles is $\mathbb{A}_{F}$. Let $\mathbf{P}=\mathbf{M N}$ be a maximal parabolic subgroup of $\mathbf{G}$, containing a fixed $F$-Borel subgroup $\mathbf{B}$ of $\mathbf{G}$. Let $\pi$ be a globally generic (cf. Section 2 here and $\left[\right.$ Sh2] ) cuspidal representation of $M=\mathbf{M}\left(\mathbb{A}_{F}\right)$. Decompose the adjoint action $r$ of ${ }^{L} M$, the $L$-group of $\mathbf{M}$, on the complex Lie algebra ${ }^{L} \mathfrak{n}$ of $L_{N}$, the $L$-group of $\mathbf{N}$ in the sense of [B], as $r=\bigoplus_{i=1}^{m} r_{i}$, ordered as in Section 1 . Let $L\left(s, \pi, r_{i}\right), s \in \mathbb{C}$, be the completed automorphic $L$-function attached to $\pi$ and $r_{i}$, where local factors at ramified places are defined as in [Sh1]. We use the same local $L$-functions (and root numbers) to normalize the local standard intertwining operators (Section 2).

Given a closed real interval $I$ and a real number $\varepsilon>0$, we let $T_{\varepsilon, I}$ be the set of all $z \in \mathbb{C}$ for which $\operatorname{Re}(z) \in I$ and $|\operatorname{Im}(z)| \geq \varepsilon$. For each $i$, there exists $\varepsilon>0$ such that $L\left(s, \pi, r_{i}\right)$ is holomorphic for $s$ with $|\operatorname{Im}(s)| \geq \varepsilon$. The main result of this paper, Theorem 4.1, its Corollary, and Proposition 3.2, states:

Theorem. Assume all the relevant local normalized operators are non-zero and holomorphic for $\operatorname{Re}(s) \geq 1 / 2$. (See Assumption 2.1 and the statement of Theorem 4.1.) Then each $L\left(s, \pi, r_{i}\right), 1 \leq i \leq m$, has a finite number of poles on $\mathbb{C}$, and there exists $\varepsilon>0$ such that each $L\left(s, \pi, r_{i}\right)$ is bounded in every $T_{\varepsilon, I}$. Consequently

Received by the editors December 21, 1999 and, in revised form, June 14, 2000.

2000 Mathematics Subject Classification. Primary 11F70, 11R42.

The first author was partially supported by a Minerva Grant.

The second author was partially supported by NSF Grant DMS-9970156. 
each $L\left(s, \pi, r_{i}\right)$ defines a function of order 1 in all of $\mathbb{C}$ (away from its poles), and a similar statement is true about partial L-functions.

There are a number of very important cases where the local assumption is already verified. We refer to Corollaries 4.2 through 4.5 for detailed statements and references to related results in each case. For example, if we let $\mathbf{G}$ be either an exceptional group of type $G_{2}$ (cf. [KSh1]), or a simply connected group of type $D_{4}$ (cf. [KSh2]), we have (Corollaries 4.3 and 4.4):

Corollary 1. Let $\pi, \pi^{\prime}$, and $\pi^{\prime \prime}$ be three cuspidal representations of $G L_{2}\left(\mathbb{A}_{F}\right)$. Assume $\pi$ is not monomial. Then the L-functions

$$
L\left(s, \pi, \operatorname{Sym}^{3}\left(\rho_{2}\right)\right) \text { and } L\left(s, \pi \times \pi^{\prime} \times \pi^{\prime \prime}\right)
$$

are bounded in every vertical strip of finite width.

In both cases $m=2$ and the second $L$-functions, i.e., for $i=2$, are Hecke $L$-functions attached to certain grössencharacters.

We remark that in the case of $L\left(s, \pi \times \pi^{\prime} \times \pi^{\prime \prime}\right)$ the corollary was originally proved in [ Ra], using the Rankin-Selberg method.

Next, applying our theorem to maximal parabolic subgroups of classical groups or general linear groups, we get (Corollaries 4.2 and 4.5):

Corollary 2. Let $\sigma$ and $\tau$ be globally generic cuspidal representations of $G L_{n}\left(\mathbb{A}_{F}\right)$ (automatically true for $\sigma$ ) and $\mathbf{G}\left(\mathbb{A}_{F}\right)$, where $\mathbf{G}$ is either $G L_{p}$ or a split classical group. Then the $L$-functions $L(s, \sigma \times \tau), L\left(s, \sigma, \Lambda^{2} \rho_{n}\right)$, and $L\left(s, \sigma, \operatorname{Sym}^{2}\left(\rho_{n}\right)\right)$ all have only a finite number of poles on $\mathbb{C}$ and there exists $\varepsilon>0$ such that they are all bounded in each $T_{\varepsilon, I}$.

It is needless to say that such results are quite important and absolutely necessary in establishing liftings of automorphic forms using converse theorems (CPS1, CPS3], CPS4, Rag1, Rag2]). In fact, recent striking developments (CKPSS, K3], [KSh4]), establishing important cases of functoriability, all rely in a fundamental way on our main Theorem 4.1. In particular, in KSh4 one needs to employ the full power of our main theorem, since, to apply the converse theorem, one now has to deal with exceptional groups of type $E_{6}$ and $E_{7}$ with $m=3$ and 4 , respectively. We again refer to the discussions before and after each of the Corollaries 4.2 through 4.5 mentioned before. One hopes that there will be other applications for such results as in [A4], [RS].

As usual, we use the theory of Eisenstein series to prove the theorem $([\overline{\mathrm{L} 2}],[\mathrm{HC}$, [MW1]). We exploit the boundedness of constant terms in each $T_{\varepsilon, I}$ with $I \geq 0$ (Lemma 101 of [ $\mathrm{HC}]$ or page 242 in [L3]). If $m=1$, granting the functional equation (2.7), Assumption 2.1, and analytic properties of matrix coefficients of normalized operators (cf. [A1], Si], Sh1]), this immediately implies the boundedness in each $T_{\varepsilon, I}$.

The general case is then inductively reduced to this case. We refer to Proposition 3.1 (cf. [Sh1], [Sh2], and [A4]) for the general induction involved.

It is here, i.e. when $m>1$, that one needs to deal with the reciprocals of $L_{-}$ functions for $\operatorname{Re}(s) \geq 1$. For that, one appeals to a non-constant Fourier coefficient of the corresponding Eisenstein series which up to a product of values of local Whittaker functions equals $\prod_{i=1}^{m} L_{S}\left(1+i s, \pi, \tilde{r}_{i}\right)^{-1}$, where $S$ is a finite set of places 
of $F$ including all the ramified and archimedean ones Sh2]. One then needs to solve two analytic problems.

The first is to estimate non-constant Fourier coefficients of a cuspidal Eisenstein series. As was pointed out to us by Erez Lapid, one cannot expect to prove, within the existing techniques, that these coefficients are of finite order in the closed half plane $\operatorname{Re}(s) \geq 0$. In fact, to estimate them, one needs to study Langlands inner product formula ([A3], [L3]) for Arthur's truncated ([A2], [A3]) Eisenstein series. A simple calculation shows that one needs to deal with the logarithmic derivative of $M(s)$, the global intertwining operator, as one approaches the unitary axis $\operatorname{Re}(s)=0$. Therefore, within our present state of knowledge, the best that one can expect is that a non-constant Fourier coefficient is given as a ratio of two analytic functions of finite order in the closed half plane $\operatorname{Re}(s) \geq 0$. We prove this in Section 6, using Muller's $[\mathrm{Mu}$ results on analytic properties of $M(s)$ (Proposition 5.4). We would like to thank Erez Lapid for his observation and several useful communications.

Each of our $L$-functions can now be expressed, through induction, as a ratio of two analytic functions of finite order in the closed half plane $\operatorname{Re}(s) \geq 1 / 2$. These quotients are obtained, not only from Fourier coefficients as explained earlier, but since we must also divide by values of Whittaker functions at archimedean places (Proposition 5.2 and Lemma 5.6).

Here is where the second problem shows up. In fact, to show that our $L$-functions are of finite order in vertical strips of finite width, to which we can now apply Phragmén-Lindelöf's theorem, we are led to use a fairly deep result from complex analysis which, strikingly enough, has itself been originally motivated by operator theory (Matsaev's theorem). Using Carleman and Poisson formulas in half disks we get an estimate for $\log \left|L_{0}\left(s, \pi, r_{i}\right)\right|$ in the open half plane $\operatorname{Re}(s)>1 / 2$ of the form

$$
\log \left|L_{0}\left(s, \pi, r_{i}\right)\right| \leq \frac{C}{\cos \theta}\left(r^{\rho}+1\right),
$$

$s-(1 / 2)=r e^{i \theta},|\theta|<\pi / 2$, for some $\rho>1$ and $C>0$, which using the functional equation we then extend to all of $\mathbb{C}$ with $|\cos \theta|$ in the denominator, but with possibly a larger $C$. To this we then apply the main theorem in the proof of Matsaev's theorem (Theorem 3, lecture 26 of [Le]) to conclude that $L\left(s, \pi, r_{i}\right)$ is indeed of finite order in $\mathbb{C}$ (away from its poles). Here $L_{0}\left(s, \pi, r_{i}\right)$ is the entire function $P_{i}(s) L\left(s, \pi, r_{i}\right)$, where $P_{i}(s)$ is the monic polynomial which gives the poles of $L\left(s, \pi, r_{i}\right)$ (cf. Sections 4 and 5 ).

This line of argument evolved from a large number of communications and conversations that we had with Misha Sodin. His ideas were communicated to us through several letters which we have included partly here as our Theorem 5.7. We are indebted to him for the countless hours that he has spent to help us with this problem.

Although the problem of boundedness in vertical strips for each $L\left(s, \pi, r_{i}\right)$ is now resolved, the much harder question of finiteness of order of each $L\left(s, \pi, r_{i}\right)^{-1}$ for $\operatorname{Re}(s) \geq 1$ still remains open which we state here as:

Conjecture. Each $L\left(s, \pi, r_{i}\right)^{-1}$ is of finite order in $\operatorname{Re}(s) \geq 1$.

Remarks are now in order on Assumption 2.1, a result which beside its applications here and elsewhere ([KSh3], [K1], [K2]), is necessary in analysis of the residual spectrum for reductive groups. (One needs even the stronger result of validity of 
Assumption 2.1 for $\operatorname{Re}(s) \geq 0$ as opposed to $\operatorname{Re}(s) \geq 1 / 2$.) In recent years, significant progress has been made towards its resolution, mainly due to local work of several mathematicians ([CSh], [K1], [K2], KSh1, [KSh2], M1], M2], [MSh, [MW2, [Z], [Zh]) and we would not be surprised if it is resolved within the next few years. One only needs to address the problem when the group is either exceptional of type different from $G_{2}$, for which the problem has already been resolved in [KSh1], or is a spin group, i.e. a simply connected covering of a special orthogonal group. We again refer to the discussions after and before each of the Corollaries 4.2 through 4.5 for appropriate credits and references. Significant progress has been recently made by Henry Kim on proving the assumption in general.

Due to the large number of equations in this paper, we were advised to explain how they are numbered. Equations in the main body of each section are given by two numbers, that of the section, followed by the number of the equation in it, e.g. equation $(5.2),(5.7),(6.12)$. On the other hand, the equations which appear in the statements and proofs of lemmas and propositions are numbered by the proposition (or lemma) number, followed by that of the equation, e.g. (5.2.1) or (5.1.2), which refer to Proposition 5.2 and Lemma 5.1, respectively.

Besides expressing their gratitude to Erez Lapid and Misha Sodin, the authors would like to thank Armand Borel for his important advice on the material in Section 6 (see the first paragraph there), as well as James Cogdell, David Drasin, Ravi Raghunathan, and Dinakar Ramakrishnan for useful discussions.

\section{Preliminaries and notation}

Let $F$ be a number field. For every place $v$ of $F$, let $F_{v}$ be the completion of $F$ at $v$. Denote by $O_{v}$ and $P_{v}$ the ring of integers of $F_{v}$ and its maximal ideal, respectively. Let $q_{v}$ be the number of elements in the residue field $O_{v} / P_{v}$. Fix a uniformizing parameter $\varpi_{v} \in P_{v}$, i.e. $P_{v}=\left(\varpi_{v}\right)$. Let ||$_{v}$ be the normalized absolute value of $F_{v}$ for which $\left|\varpi_{v}\right|_{v}=q_{v}^{-1}$. Finally, denote by $\mathbb{A}_{F}$ the ring of adeles of $F$. If $\mathbb{I}_{F}$ is the group of ideles of $F$, the units of $\mathbb{A}_{F}$, then $\mathbb{I}_{F}=\mathbb{I}_{F}^{1} \times \mathbb{R}_{+}^{*}$, where $\mathbb{I}_{F}^{1}$ is the subgroup of ideles of norm one, while $\mathbb{R}_{+}^{*}$ denotes the multiplicative group of positive real numbers.

Let $\mathbf{G}$ be a quasisplit connected reductive algebraic group over $F$. Fix a Borel subgroup $\mathbf{B}$ of $\mathbf{G}$ defined over $F$. Write $\mathbf{B}=\mathbf{T} \mathbf{U}$ where $\mathbf{T}$ is a maximal torus of $\mathbf{G}$ and $\mathbf{U}$ denotes the unipotent radical of $\mathbf{B}$, both over $F$. Let $\mathbf{P}$ be an $F$-parabolic subgroup of $\mathbf{G}$ with $\mathbf{P} \supset \mathbf{B}$. Let $\mathbf{P}=\mathbf{M N}, \mathbf{N} \subset \mathbf{U}$, be a Levi decomposition of $\mathbf{P}$. We will fix $\mathbf{M}$ by assuming $\mathbf{M} \supset \mathbf{T}$.

For every place $v, \mathbf{G}$ may be considered as a group over $F_{v}$. We then set $G_{v}=\mathbf{G}\left(F_{v}\right)$. We similarly have $B_{v}, T_{v}, U_{v}, P_{v}, M_{v}$, and $N_{v}$. Finally, set $G=$ $\mathbf{G}\left(\mathbb{A}_{F}\right), B=\mathbf{B}\left(\mathbb{A}_{F}\right)$, etc.

For almost all places $v$, where $\mathbf{G}$ is defined over $O_{v}$, we let $K_{v}=\mathbf{G}\left(O_{v}\right)$. Otherwise, we fix a special maximal compact subgroup $K_{v} \subset G_{v}$ which we will further assume to be adapted to $A_{0, v}=\mathbf{A}_{0}\left(F_{v}\right)$, where $\mathbf{A}_{0}$ is the maximal $F$-split torus in T. Set $K=\otimes_{v} K_{v}$. Then $G=P K$.

Let $\mathbf{A}$ be the split component of the center of $\mathbf{M}$. Then $\mathbf{A} \subset \mathbf{A}_{0} \subset \mathbf{T}$. For every $F$-group $\mathbf{H}$, let $X(\mathbf{H})_{F}$ be the group of $F$-rational characters of $\mathbf{H}$. Set $\mathfrak{a}=\operatorname{Hom}\left(X(\mathbf{M})_{F}, \mathbb{R}\right)$, the real Lie algebra of $\mathbf{A}$. Then

$$
\mathfrak{a}^{*}=X(\mathbf{M})_{F} \otimes_{\mathbb{Z}} \mathbb{R}=X(\mathbf{A})_{F} \otimes_{\mathbb{Z}} \mathbb{R}
$$

and $\mathfrak{a}_{\mathbb{C}}^{*}=\mathfrak{a}^{*} \otimes_{\mathbb{R}} \mathbb{C}$ is the complex dual of $\mathfrak{a}$. 
The embedding $X(\mathbf{M})_{F} \subset X(\mathbf{M})_{F_{v}}$ induces a map $\mathfrak{a}_{v} \rightarrow \mathfrak{a}$ (mistakenly called an embedding in $\underline{\text { Sh2 }]})$, where $\mathfrak{a}_{v}=\operatorname{Hom}\left(X(\mathbf{M})_{F_{v}}, \mathbb{R}\right)$. There exists a homomorphism

$$
H_{M}: M \rightarrow \mathfrak{a}
$$

defined by

$$
\exp \left\langle\chi, H_{M}(m)\right\rangle=\prod_{v}\left|\chi\left(m_{v}\right)\right|_{v}
$$

for all $\chi \in X(\mathbf{M})_{F}$ and $m=\left(m_{v}\right)$. Extend $H_{M}$ to all of $G$ by making it trivial on $N$ and $K$ which we denote by $H_{P}$. We can define $H_{P_{v}}$ for each $v$ in precisely the same manner. Then

$$
\exp \left\langle\chi, H_{P}(m)\right\rangle=\prod_{v} \exp \left\langle\chi, H_{P_{v}}\left(m_{v}\right)\right\rangle \quad\left(\chi \in X(\mathbf{M})_{F}\right)
$$

using the embedding $X(\mathbf{M})_{F} \hookrightarrow X(\mathbf{M})_{F_{v}}$ for each $v$. Observe that given $m \in M$, this is only a finite product.

Let $M^{1}$ be the kernel of $H_{M}$. Then $M$ is the direct product of $M^{1}$ and $A_{\infty}^{0}$, where $A_{\infty}^{0}$ is the connected component of identity in $A_{\infty}=\prod_{v \mid \infty} A_{v}, A_{v}=\mathbf{A}\left(F_{v}\right)$.

Denote by $\Sigma$ the set of $F$-roots of $\mathbf{A}_{0}$. Then $\Sigma=\Sigma^{+} \cup \Sigma^{-}$, where $\Sigma^{+}$is the set of positive roots, i.e. those generating $\mathbf{U}$. Let $\Delta \subset \Sigma^{+}$be the set of simple roots. We shall identify the roots of $\mathbf{A}$ in $\mathbf{N}$ with a subset of $\Sigma^{+}$.

We shall say $\mathbf{P}$ is maximal if the roots of $\mathbf{A}$ in $\mathbf{N}$, as identified with a subset of $\Sigma^{+}$, contain a single simple root $\alpha \in \Delta$. Thus $\alpha$ denotes the unique reduced root of $\mathbf{A}$ in $\mathbf{N}$. Throughout this paper we shall assume $\mathbf{P}$ is maximal. Let $\rho_{\mathbf{P}}$ be half the sum of roots generating the Lie algebra of $\mathbf{N}$. Then $\tilde{\alpha}=\left\langle\rho_{\mathbf{P}}, \alpha\right\rangle^{-1} \rho_{\mathbf{P}}$ belongs to $\mathfrak{a}^{*}$. We refer to Section 1 of $\underline{S h 2}$ for the definition of $\langle$,$\rangle in terms of the Killing form$ for non-restricted roots of $\mathbf{T}$ in $\mathbf{U}$, restricting to $\Sigma^{+}$. Observe that, for each $v, \tilde{\alpha}$ can be realized as an element in $\mathfrak{a}_{v}^{*}$ through the embedding $\mathfrak{a}^{*} \hookrightarrow \mathfrak{a}_{v}^{*}$. From now on, we shall identify $\mathbb{C}$ with a subspace of $\mathfrak{a}_{\mathbb{C}}^{*}$ by identifying $s \in \mathbb{C}$ with $s \tilde{\alpha} \in \mathfrak{a}_{\mathbb{C}}^{*}$.

For every connected reductive group $\mathbf{H}$ over $F$, let ${ }^{L} H$ be its $L$-group $[\mathbb{B}]$. We use $\hat{H}$ to denote ${ }^{L} H^{0}$, the connected component of 1 in ${ }^{L} H$. For each $v$, there is a natural homomorphism ${ }^{L} H_{v} \rightarrow{ }^{L} H$, where ${ }^{L} H_{v}$ is the $L$-group of $\mathbf{H}$ as a group over $F_{v}$. Let $\eta_{v}:{ }^{L} M_{v} \rightarrow{ }^{L} M$ be this map for $\mathbf{M}$. Given a finite dimensional complex representation $r$ of ${ }^{L} M$, the representation $r_{v}=r \cdot \eta_{v}$ becomes one of ${ }^{L} M_{v}$. We define ${ }^{L} N$, the $L$-group of $\mathbf{N}$, as in the sense of $[\mathrm{B}$.

Let ${ }^{L} \mathfrak{n}$ be the (complex) Lie algebra of ${ }^{L} N$. The group ${ }^{L} M$ acts on ${ }^{L} \mathfrak{n}$ by adjoint action $r$. We decompose $r=\bigoplus_{i=1}^{m} r_{i}$ to its irreducible constituents on ${ }^{L} \mathfrak{n}=V=\bigoplus_{i=1}^{m} V_{i}$ and index them according to an upper central series of ${ }^{L} \mathfrak{n}$. More precisely, $m$ is equal to the nilpotence class of ${ }^{L} N$ (or ${ }^{L} \mathfrak{n}$ ), and each $r_{i}$ corresponds to a factor in the series with $r_{m}$ corresponding to the action on the center $V_{m}$ of ${ }^{L_{\mathfrak{n}}}$, and then increasing up on the series. Notice that

$$
V_{i}=\left\{X_{\beta^{v}} \in{ }^{L} \mathfrak{n} \mid\langle\tilde{\alpha}, \beta\rangle=i\right\}
$$

and therefore the order is that of the increasing eigenvalues of the action of ${ }^{L} A$ on $L_{\mathfrak{n}}$. 


\section{2. $L$-FUNCTIONS AND INTERTWINING OPERATORS}

Let $\pi=\otimes_{v} \pi_{v}$ be a cuspidal representation of $M=\mathbf{M}\left(\mathbb{A}_{F}\right)$. For our purposes, we may and will assume that $\pi$ is trivial on $A_{\infty}^{0}$ and therefore is one of $M^{1}$, the kernel of $H_{M}$.

Let $W$ be the Weyl group of $\mathbf{A}_{0}$ in $\mathbf{G}$. We use $\theta$ to denote the subset of $\Delta$ which generated $\mathbf{M}$. Then $\Delta=\theta \cup\{\alpha\}$. There exists a unique element $\tilde{w} \in W$ satisfying $\tilde{w}(\theta) \subset \Delta$ but $\tilde{w}(\alpha) \in \Sigma^{-}$. Fix a representative $w \in K \cap \mathbf{G}(F)$ for $\tilde{w}$.

Let $I(s, \pi)$ be the induced representation

$$
I(s, \pi)=\operatorname{Ind}_{M N \uparrow G} \pi \otimes \exp \left\langle s, H_{P}()\right\rangle \otimes \mathbf{1},
$$

where we have identified $\mathbb{C}$ with a subspace of $\mathfrak{a}_{\mathbb{C}}^{*}$ by $s \mapsto s \tilde{\alpha}$ as in Section 1 . Then

$$
I\left(s, \pi_{v}\right)=\underset{M_{v} N_{v} \uparrow G_{v}}{\operatorname{Ind}} \pi_{v} \otimes \exp \left\langle s, H_{P_{v}}()\right\rangle \otimes \mathbf{1} .
$$

Let $\mathbf{M}^{\prime}$ be the Levi subgroup of $\mathbf{G}$ with $\mathbf{M}^{\prime} \supset \mathbf{T}$ and generated by $\tilde{w}(\theta)$. Let $\mathbf{P}^{\prime} \supset \mathbf{B}$ be the parabolic subgroup of $\mathbf{G}$ which has $\mathbf{M}^{\prime}$ as a Levi factor. Let $\mathbf{N}^{\prime} \subset \mathbf{U}$ be its unipotent radical. Given $f$ in the space $V(s, \pi)$ of $I(s, \pi)$ and $\operatorname{Re}(s)$ sufficiently large, define

$$
M(s, \pi) f(g)=\int_{N^{\prime}} f\left(w^{-1} n g\right) d n \quad(g \in G) .
$$

Observe that if $f=\otimes_{v} f_{v}$, then for almost all $v, f_{v}$ is the unique $K_{v}$-fixed function normalized by $f_{v}\left(e_{v}\right)=1$. Finally, for each $v$, let $M\left(s, \pi_{v}, w\right)=M\left(s \tilde{\alpha}, \pi_{v}, w\right)$ be the corresponding local intertwining operator defined for $\operatorname{Re}(s)$ sufficiently large by

$$
M\left(s \tilde{\alpha}, \pi_{v}, w\right) f_{v}(g)=\int_{N_{v}^{\prime}} f_{v}\left(w^{-1} n g\right) d n \quad\left(g \in G_{v}\right)
$$

Then

$$
M(s, \pi)=\otimes_{v} M\left(s \tilde{\alpha}, \pi_{v}, w\right) .
$$

Suppose $v$ is such that both $\mathbf{G}$ and $\pi_{v}$ are unramified. If $\tau_{v}$ is the Frobenius conjugacy class in $\operatorname{Gal}\left(L_{w} / F_{v}\right), w \mid v$, where $L$ is a finite Galois extension of $F$ over which $\mathbf{G}$ splits, then there exists $A_{v} \in \hat{T}={ }^{L} T^{0}$ such that the ${ }^{L} M$-conjugacy class of $A_{v} \rtimes \tau_{v}$ determines the class of $\pi_{v}$ uniquely (cf. [B], Sh2]). We may and will assume that $A_{v}$ is fixed by $\tau_{v}$. The local Langlands $L$-function attached to $\pi_{v}$ and $r_{v}$ is then defined by

$$
L\left(s, \pi_{v}, r_{v}\right)=\operatorname{det}\left(I-r_{v}\left(A_{v} \rtimes \tau_{v}\right) q_{v}^{-s}\right)^{-1} .
$$

Let $S$ be a finite set of places of $F$ such that for every $v \notin S, \pi_{v}$ and $\mathbf{G}$ are both unramified. Set

$$
L_{S}(s, \pi, r)=\prod_{v \notin S} L\left(s, \pi_{v}, r_{v}\right) .
$$

Now, for each $i, 1 \leq i \leq m$, let $r_{i}$ be as before the adjoint action of ${ }^{L} M$ on $V_{i},{ }^{L} \mathfrak{n}=\bigoplus_{i=1}^{m} V_{i}$. It is well known (cf. [L1], Sh2]) that

$$
M(s, \pi) f=\otimes_{v \in S} M\left(s \tilde{\alpha}, \pi_{v}, w\right) f_{v} \otimes_{v \notin S} \tilde{f}_{v} \cdot \prod_{i=1}^{m} L_{S}\left(i s, \pi, \tilde{r}_{i}\right) / L_{S}\left(1+i s, \pi, \tilde{r}_{i}\right),
$$


where $f=\otimes_{v} f_{v}$ is such that for each $v \notin S, f_{v}$ is the unique $K_{v}$-fixed function normalized by $f_{v}\left(e_{v}\right)=1$ and for each $i, \tilde{r}_{i}$ is the contragredient of $r_{i}, i=1, \ldots, m$. Here $\tilde{f}_{v}$ is the $K_{v}$-fixed function in the space of $I\left(-s, w\left(\pi_{v}\right)\right)$ normalized the same way.

Let $\psi=\otimes_{v} \psi_{v}$ be a non-trivial character of $F \backslash \mathbb{A}_{F}$. Let $\chi$ be a non-degenerate character of $\mathbf{U}(F) \backslash U$, defined by $\psi$ and compatible with $\tilde{w}$ (cf. [Sh1]). If $\mathbf{U}^{0}=$ $\mathbf{U} \cap \mathbf{M}$, we will use $\chi$ to also denote $\chi \mid U^{0}$. From now on, we shall assume $\pi$ is $\chi$-generic in the sense that there exists a cusp form $\varphi$ in the space of $\pi$ with a non-zero $\chi$-Fourier coefficient (cf. Section 3 of [Sh2]).

We shall now concentrate on places in $S$. We assume $S$ is large enough so that for $v \notin S$ each $\psi_{v}$ and $\chi_{v}$ is unramified. For each $v$, in Sh1], one defines a root number $\varepsilon\left(s, \pi_{v}, r_{i, v}, \psi_{v}\right)$ and an $L$-function $L\left(s, \pi_{v}, r_{i, v}\right)$, and normalizes $M\left(s \tilde{\alpha}, \pi_{v}, w\right)$ as

$$
\begin{aligned}
& N\left(s \tilde{\alpha}, \pi_{v}, w\right)= \\
& \prod_{i=1}^{m} \varepsilon\left(i s, \pi_{v}, \tilde{r}_{i, v}, \psi_{v}\right) L\left(1+i s, \pi_{v}, \tilde{r}_{i, v}\right) L\left(i s, \pi_{v}, \tilde{r}_{i, v}\right)^{-1} M\left(s \tilde{\alpha}, \pi_{v}, w\right) .
\end{aligned}
$$

Then

$$
N\left(s \tilde{\alpha}, \pi_{v}, w\right) N\left(s w(\tilde{\alpha}), w\left(\pi_{v}\right), w^{-1}\right)=\mathbf{1} .
$$

Here we have fixed the representative $w$ of $\tilde{w}$ as in [Sh3], and we understand that in the normalizing factor for $N\left(s w(\tilde{\alpha}), w\left(\pi_{v}\right), w^{-1}\right), \psi_{v}$ is replaced with $\bar{\psi}_{v}$.

The map $f_{v}^{0}(g) \mapsto \exp \left\langle s \tilde{\alpha}, H_{P_{v}}(g)\right\rangle f_{v}^{0}(g), g \in G_{v}$, defines a bijection from $V\left(0, \pi_{v}\right)$ onto $V\left(s, \pi_{v}\right)$. Set $f_{v}(g)=\exp \left\langle s \tilde{\alpha}, H_{P_{v}}(g)\right\rangle f_{v}^{0}(g)$. We make the following assumption.

Assumption 2.1. Fix a place $v$ and assume $\operatorname{Re}(s) \geq 1 / 2$. Then there exists a function $f_{v}^{0} \in V\left(0, \pi_{v}\right)$ such that $N\left(s \tilde{\alpha}, \pi_{v}, w\right) f_{v}(g)$ is non-zero and holomorphic at $s$ for some $g \in G_{v}$.

The assumption is quite natural and is already verified in many cases including $G L(n)$ MW2 and a number of other groups (K1], K2], KSh1, KSh2]). We will comment more on it in Section 4, where we discuss a number of important applications. One expects the validity of Assumption 2.1 even for $\operatorname{Re}(s) \geq 0$ which is necessary for the study of residual spectrums. But this is usually harder to prove.

Next, as in Sh1], we let

$$
L\left(s, \pi, r_{i}\right)=\prod_{v} L\left(s, \pi_{v}, r_{i, v}\right)
$$

and

$$
\varepsilon\left(s, \pi, r_{i}\right)=\prod_{v} \varepsilon\left(s, \pi_{v}, r_{i, v}, \psi_{v}\right) .
$$

Then the functional equations (cf. [Sh1], Sh4])

$$
L\left(s, \pi, r_{i}\right)=\varepsilon\left(s, \pi, r_{i}\right) L\left(1-s, \pi, \tilde{r}_{i}\right) \quad(1 \leq i \leq m)
$$

allow us to restrict ourselves to proofs of analytic properties of these $L$-functions only for $\operatorname{Re}(s) \geq 1 / 2$. 


\section{INDUCTION AND FINITENESS OF POLES}

Assumption 2.1 allows us to prove that each $L\left(s, \pi, r_{i}\right)$ has only a finite number of poles on all of $\mathbb{C}$. While many cases of this result for $L_{S}\left(s, \pi, r_{i}\right)$ were proved in [Sh2], Sh5] with no assumptions, the general case is still not in hand unless Assumption 2.1 is used. We start by recalling the general induction ([Sh1], Sh2]) which is needed in order to answer different questions about each individual $L\left(s, \pi, r_{i}\right)$ using this approach. As observed by Arthur A4, our induction is endoscopic. For the sake of completeness, we include a result which captures the main induction step of [Sh1] (cf. Propositions 4.1, 4.2, 5.1, Theorem 3.5 of [Sh1]).

Proposition 3.1. Let $F$ be a number field (a local field of characteristic zero, respectively). Let $\mathbf{G}$ be a quasisplit connected reductive group over $F$. Let $\mathbf{P}=\mathbf{M N}$ be a standard maximal parabolic subgroup of $\mathbf{G}$ with respect to a $F$-Borel subgroup B as before. Let $\pi$ be a globally generic cuspidal representation of $M=\mathbf{M}\left(\mathbb{A}_{F}\right)$ (irreducible admissible generic representation of $M=\mathbf{M}(F)$, respectively). Let $r=\bigoplus_{i=1}^{m} r_{i}$ be the adjoint action of ${ }^{L} M$ on ${ }^{L} \mathfrak{n}$ as before. Then for each $i, 2 \leq i \leq m$, there exist a quasisplit connected reductive $F$-group $\mathbf{G}_{i}$, a maximal $F$-parabolic subgroup $\mathbf{P}_{i}=\mathbf{M}_{i} \mathbf{N}_{i}$ of $\mathbf{G}_{i}$, and a globally generic cuspidal representation $\pi^{\prime}$ of $M_{i}=\mathbf{M}_{i}\left(\mathbb{A}_{F}\right)$ (an irreducible admissible generic representation $\pi^{\prime}$ of $M_{i}=\mathbf{M}_{i}(F)$, respectively) such that, if the adjoint action $r^{\prime}$ of ${ }^{L} M_{i}$ on ${ }^{L} \mathfrak{n}_{i}$ decomposes as $r^{\prime}=$ $m^{\prime}$ $\bigoplus_{j=1} r_{j}^{\prime}$, then

$$
L\left(s, \pi, r_{i}\right)=L\left(s, \pi^{\prime}, r_{1}^{\prime}\right)
$$

and

$$
\varepsilon\left(s, \pi, r_{i}\right)=\varepsilon\left(s, \pi^{\prime}, r_{1}^{\prime}\right)
$$

$\left(\varepsilon\left(s, \pi, r_{i}, \psi_{F}\right)=\varepsilon\left(s, \pi^{\prime}, r_{1}^{\prime}, \psi_{F}\right)\right.$, respectively). Moreover $m^{\prime}<m$ and for $F$ a number field if the data outside $S$ is unramified for $(\mathbf{G}, \mathbf{M}, \pi)$, then the same is true for each $\left(\mathbf{G}_{i}, \mathbf{M}_{i}, \pi^{\prime}\right)$.

Remark. Arthur A4 has shown that groups $\mathbf{G}_{i}$ can be taken to be among the elliptic endoscopic groups of $\mathbf{G}$.

Proposition 3.2. Let $F$ be a number field and $\pi$ a globally generic cuspidal representation of $M^{1}=\mathbf{M}\left(\mathbb{A}_{F}\right)^{1}$. With notation and terminology as in Section 2 and this section, suppose for each $v \in S$, Assumption 2.1 holds for $\left(G_{v}, M_{v}, \pi_{v}\right)$, and each $\left(G_{i, v}, M_{i, v}, \pi_{v}^{\prime}\right)$, as well as for those groups attached to them, and so on, via induction of Proposition 3.1. Then each $L\left(s, \pi, r_{i}\right)$ has only a finite number of poles on all of $\mathbb{C}$. They are all real.

Corollary 3.3. Under the above assumption, each $L_{S}\left(s, \pi, r_{i}\right)$ extends to a meromorphic function of $s$ on all of $\mathbb{C}$ with only a finite number of poles. They are all real.

Proof of Proposition 3.2. We shall reformulate equation (2.4) as

$$
M(s, \pi) f=\prod_{i=1}^{m} \varepsilon\left(i s, \pi, \tilde{r}_{i}\right)^{-1} L\left(i s, \pi, \tilde{r}_{i}\right) L\left(1+i s, \pi, \tilde{r}_{i}\right)^{-1} \otimes_{v} N\left(s \tilde{\alpha}, \pi_{v}, w\right) f_{v} .
$$


Under our assumption (Assumption 2.1), the finiteness of poles for $M(s, \pi)$ for $s>0$ (cf. [L2], $\mathrm{HC}$ ) implies the same for

$$
\prod_{i=1}^{m} L\left(i s, \pi, \tilde{r}_{i}\right) / L\left(1+i s, \pi, \tilde{r}_{i}\right)
$$

for $\operatorname{Re}(s) \geq 1 / 2$. By $(2.7)$ it is enough to prove the finiteness of poles for each $L\left(s, \pi, r_{i}\right)$ only for $\operatorname{Re}(s) \geq 1 / 2$. We do this by induction on $m$.

By Lemma 5.6 of [Sh2], applied to the completed $L$-functions rather than the $L_{S}$ 's, which by the nature of local $L$-functions is still valid,

$$
\prod_{i=1}^{m} L\left(1+i s, \pi, \tilde{r}_{i}\right)
$$

has only a finite number of (simple) zeros for $\operatorname{Re}(s) \geq 0$.

Our induction hypothesis now becomes: Suppose $i \geq 2$. Then each $L\left(s, \pi, r_{i}\right)$ has only a finite number of poles for $\operatorname{Re}(s) \geq \frac{1}{2}$ and zeros for $\operatorname{Re}(s) \geq 1$, respectively.

Now, suppose $L\left(s, \pi, r_{i}\right)$ has only a finite number of poles and zeros for $\operatorname{Re}(s) \geq$ $1 / 2$ and $\operatorname{Re}(s) \geq 1$, respectively, for $2 \leq i \leq m$. Then it follows from applying (3.3.3) to $\tilde{\pi}$, the contragredient of $\pi$, that $L\left(s, \pi, r_{1}\right)$ also has only a finite number of zeros for $\operatorname{Re}(s) \geq 1$.

The finiteness of poles for $\operatorname{Re}(s) \geq 1 / 2$ for $L\left(s, \pi, r_{1}\right)$ now follows from finiteness of poles for (3.3.2) applied to $\tilde{\pi}$ over the same range and our induction hypothesis for $2 \leq i \leq m$. This completes the proof.

Corollary 3.4. Under the assumption of Proposition 3.2 each $L\left(s, \pi, r_{i}\right)$ has only a finite number of zeros for $\operatorname{Re}(s) \geq 1$. They are all real.

\section{BOUNDEDNESS IN VERTICAL STRIPS}

Given a real number $\varepsilon>0$ and a real closed finite integral $I$, let

$$
T_{\varepsilon, I}=\{s \in \mathbb{C}|| \operatorname{Im}(s) \mid \geq \varepsilon, \operatorname{Re}(s) \in I\},
$$

which we call a pair of "half strips of finite width".

A meromorphic function $f(s), s \in \mathbb{C}$, is said to be bounded in vertical strips of finite width or simply in finite vertical strips if for each $I$, there exists an $\varepsilon>0$ such that $f$ is bounded in $T_{\varepsilon, I}$. In particular, $f$ will have to be holomorphic on $T_{\varepsilon, I}$. When $f$ is entire this definition agrees with the usual boundedness in finite vertical strips.

By Proposition 3.2, for each $i, 1 \leq i \leq m, L\left(s, \pi, r_{i}\right)$ is meromorphic with at most a finite number of poles on $\mathbb{C}$. The main result of this paper is the following theorem. We refer to [CPS1], [CPS3], [CPS4], Rag1, Rag2], Ra], CKPSS], [KSh4], [K3 for its applications in converse theorems.

Theorem 4.1. Let $F$ be a number field and $\pi$ a globally generic cuspidal representation of $M=\mathbf{M}\left(\mathbb{A}_{F}\right)$. With notation and terminology as in Sections 2 and 3, suppose for each $v \in S$, Assumption 2.1 holds for $\left(G_{v}, M_{v}, \pi_{v}\right)$ and each $\left(G_{i, v}, M_{i, v}, \pi_{v}^{\prime}\right)$, as well as for those groups attached to them, and so on, via induction of Proposition 3.1. Then each $L\left(s, \pi, r_{i}\right)$, a meromorphic function with possibly only a finite number of poles on $\mathbb{C}$, is bounded in every vertical strip of finite width. 
We shall prove Theorem 4.1 in the next few sections. But for the moment we shall state a number of results by specializing Theorem 4.1 to special cases where Assumption 2.1 is already verified. A collection of very important $L$-functions are among these which we shall now list. Each of these results is important at least when one tries to apply converse theorems to establish existence of automorphic forms and prove new cases of functoriality (cf. [CPS1], CPS3], [CPS4], [Rag1, Rag2, [Ra], CKPSS, [KSh4], K3].

For each $i, 1 \leq i \leq m$, let $s_{i}=\left\{s_{i j}\right\}$ be the set of poles of $L\left(s, \pi, r_{i}\right)$, where each pole is counted with multiplicity. By Proposition 3.2, each $s_{i}$ is finite, and every $s_{i j} \in \mathbb{R}$ if $\pi$ is a representation of $M^{1}$. Let

$$
P_{i}(s)=\prod_{j}\left(s-s_{i j}\right) .
$$

Then each $P_{i}(s) L\left(s, \pi, r_{i}\right)$ is entire. Since local $L$-functions at archimedean places are each a product of $\Gamma$-functions in linear combinations of $s$ which are consequently of order 1 in $\operatorname{Re}(s) \geq 1 / 2$, Theorem 4.1 and the functional equation immediately imply:

Corollary. For each $i$, both $P_{i}(s) L\left(s, \pi, r_{i}\right)$ and $P_{i}(s) L_{S}\left(s, \pi, r_{i}\right)$ are entire functions of order 1 on all of $\mathbb{C}$.

We start with the Rankin-Selberg product $L$-functions for $G L_{n}\left(\mathbb{A}_{F}\right) \times G L_{p}\left(\mathbb{A}_{F}\right)$, where $n$ and $p$ are two positive integers. Let $\pi$ and $\pi^{\prime}$ be cuspidal representations of $G L_{n}\left(\mathbb{A}_{F}\right)$ and $G L_{p}\left(\mathbb{A}_{F}\right)$, respectively. Recall that the local Rankin-Selberg $L-$ function attached to $\pi_{v}$ and $\pi_{v}^{\prime}$ when they are unramified and attached to $A_{v} \subset$ $G L_{n}(\mathbb{C})$ and $A_{v}^{\prime} \subset G L_{p}(\mathbb{C})$ is defined by

$$
L\left(s, \pi_{v} \times \pi_{v}^{\prime}\right)=\operatorname{det}\left(I-A_{v} \otimes A_{v}^{\prime} q_{v}^{-s}\right)^{-1} .
$$

At ramified places, the local factors are defined by either of two methods and are equal ([JPSS], Sh3], Sh6], Sh1]), and, in view of the recent proof of the local Langlands conjecture $([\mathrm{HT}, \overline{\mathrm{H}}],[\mathrm{L} 4],[\mathrm{Sh} 3])$, are all those of Artin. Moreover, the global $L$-function $L\left(s, \pi \times \pi^{\prime}\right)$ is entire unless $\pi^{\prime} \cong \tilde{\pi} \otimes|\operatorname{det}(\quad)|^{s_{0}}$ for some $s_{0} \in \mathbb{C}$ (cf. [JS1], JJS2], [MW2]) and satisfies a standard functional equation ([Sh1], [Sh3], Sh4], Sh6]). Suppose $\pi^{\prime} \cong \tilde{\pi}$. Then $L\left(s, \pi \times \pi^{\prime}\right)$ has simple poles at $s=0$ and 1 (cf. [JS2]).

In our setting $\mathbf{G}=G L_{n+p}, \mathbf{M}=G L_{n} \times G L_{p}$, and the representation of $M$ is $\pi \otimes \tilde{\pi}^{\prime}$. In this case, $m=1$ and $L\left(s, \pi \times \pi^{\prime}\right)$ is the corresponding $L$-function. Consequently $P_{1}(s)=s(s-1)$ if $\pi^{\prime} \cong \tilde{\pi}$. Our Theorem 4.1 then gives another proof of the following result which was first observed in print by Rudnick-Sarnak in [RS, to which we refer the reader for some important applications on distribution of zeros for certain $L$-functions.

Corollary 4.2. The $L$-function $s(s-1) L\left(s, \pi \times \pi^{\prime}\right)$ is bounded in every vertical strip of finite width.

Proof. We only need to verify Assumption 2.1. But a much stronger result is proved in this case by Moeglin-Waldspurger (Proposition I.10 of [MW2]).

Next we let $\pi$ be a cuspidal representation of $G L_{2}\left(\mathbb{A}_{F}\right)$. Let $\operatorname{Sym}^{3}\left(\rho_{2}\right)$ be the third symmetric cube representation of $G L_{2}(\mathbb{C})$ on symmetric tensors of rank 3 . Here $\rho_{2}=\operatorname{Sym}^{1}\left(\rho_{2}\right)$ is just the standard representation of $G L_{2}(\mathbb{C})$. Recall that if 
$\pi_{v}$ is unramified and its corresponding semisimple conjugacy class is represented by $\operatorname{diag}\left(\alpha_{v}, \beta_{v}\right) \in G L_{2}(\mathbb{C})$, then

$$
L\left(s, \pi_{v}, \operatorname{Sym}^{3}\left(\rho_{2}\right)\right)=\prod_{j=0}^{3}\left(1-\alpha_{v}^{j} \beta_{v}^{3-j} q_{v}^{-s}\right)^{-1} .
$$

At ramified places the local factors are defined in [Sh3], Sh7] and are again those of Artin attached to $\pi_{v}$ through the local Langlands conjecture (cf. $[\mathrm{HT}],[\mathrm{H}],[\mathrm{Ku}]$, [L4).

In our setting $\mathbf{G}$ is the split group of type $G_{2}$ and $\mathbf{M} \cong G L_{2}$ is generated by the long simple root of $\mathbf{G}$. Then $m=2, r_{1}=r_{3}^{0}=\operatorname{Sym}^{3}\left(\rho_{2}\right) \otimes\left(\Lambda^{2} \rho_{2}\right)^{-1}$ and $r_{2}=\Lambda^{2} \rho_{2}$. Observe that if $\omega_{\pi}$ is the central character of $\pi$, then

$$
L\left(s, \pi \otimes \omega_{\pi}, r_{1}\right)=L\left(s, \pi, \operatorname{Sym}^{3}\left(\rho_{2}\right)\right)
$$

and

$$
L\left(s, \pi, r_{2}\right)=L\left(s, \omega_{\pi}\right) .
$$

It was proved in KSh1 that $L\left(s, \pi, \operatorname{Sym}^{3}\left(\rho_{2}\right)\right)$ is entire unless $\pi$ is defined by a grossencharacter $\chi$ of a quadratic extension of $F$, i.e. $\pi$ is monomial (cf. KSh1]). Again assuming $\pi$ is a cuspidal representation of $G L_{2}\left(\mathbb{A}_{F}\right)^{1}$ which amounts to $\omega_{\pi}$ being trivial on $\mathbb{R}_{+}^{*}$ through the identification of the center of $G L_{2}\left(\mathbb{A}_{F}\right)$ with $\mathbb{I}_{F}=\mathbb{I}_{F}^{1} \times \mathbb{R}_{+}^{*}$, the poles of $L\left(s, \pi, \operatorname{Sym}^{3}\left(\rho_{2}\right)\right)$ are at $s=0,1$ and appear if and only if $\chi^{3}=1$. Thus $P_{1}(s)=s(s-1)$ in this case. We furthermore have:

Corollary 4.3. a) If $\pi$ is not monomial, then the $L$-function $L\left(s, \pi, \operatorname{Sym}^{3}\left(\rho_{2}\right)\right)$ is bounded in vertical strips of finite width. The same is true in every $T_{\varepsilon, I}$, otherwise.

b) Let $\rho=\otimes_{v} \rho_{v}$ be an arbitrary grossencharacter of $F$. Define

$$
L\left(s, \pi, r_{3}^{0}, \rho\right)=L\left(s, \pi \otimes \rho, r_{3}^{0}\right),
$$

where $r_{3}^{0}$ is the adjoint cube representation of $G L_{2}(\mathbb{C})$ defined by $r_{3}^{0}=\operatorname{Sym}^{3}\left(\rho_{2}\right) \otimes$ $\left(\Lambda^{2} \rho_{2}\right)^{-1}$. Assume $\pi$ is not monomial. Then $L\left(s, \pi, r_{3}^{0}, \rho\right)$ is bounded in vertical strips of finite width for every $\rho$.

Proof. Again Assumption 2.1 is verified already in Propositions 3.1 and 3.2 of KSh1.

Remark. Observe that part $\mathrm{b})$ is formulated so that whenever similar results about twists of $r_{3}^{0}$ with cusp forms on $G L_{2}\left(\mathbb{A}_{F}\right)$ become available, the converse theorem can be used to imply the existence of the functorial lift $r_{3, *}^{0}$ of $r_{3}^{0}$. This would be a spectacular result (cf. [GJ] for $\operatorname{Sym}^{2}\left(\rho_{2}\right)$ ). We refer to [KSh1, Sh8] for a discussion of these issues. Observe that if the functorial lift $\Pi=r_{3, *}^{0}(\pi)$ of $\pi$ exists, then $\operatorname{Sym}^{3}\left(\rho_{2}\right)_{*}(\pi)=\Pi \otimes \omega_{\pi}$. Note that boundedness in this case can also be directly proved since $L\left(s, \pi, r_{3}^{0}\right)$ is the ratio of two Rankin product $L$-functions (cf. [KSh1]), both of which are entire of finite order by Corollary 4.2. (The existence of $r_{3, *}^{0}$ is now proved in KSh4.)

Next, we turn our attention to the Rankin triple product $L$-functions a lá Garrett Ga], Piatetski-Shapiro-Rallis GPSR, Ikeda [1], Ramakrishnan [Ra, and KimShahidi KSh2]. More precisely, let $\pi, \pi^{\prime}, \pi^{\prime \prime}$ be three cuspidal representations of $G L_{2}\left(\mathbb{A}_{F}\right)$. Assume for some place $v$ that all these local components are unramified, and let $A_{v}, A_{v}^{\prime}, A_{v}^{\prime \prime}$ be the corresponding conjugacy classes in $G L_{2}(\mathbb{C})$. The local 
Langlands $L$-functions, the local Rankin triple product $L$-function, is then defined by

$$
L\left(s, \pi_{v} \times \pi_{v}^{\prime} \times \pi_{v}^{\prime \prime}\right)=\operatorname{det}\left(I-A_{v} \otimes A_{v}^{\prime} \otimes A_{v}^{\prime \prime} q_{v}^{-s}\right)^{-1} .
$$

Again, for each place $v$, ramified or otherwise, the local factors are defined by both methods and are equal to those defined through the local Langlands conjecture as Artin factors. This equality is due to Ramakrishnan [Ra]. Moreover, using either the method of Rankin-Selberg, or that of Eisenstein series, one can prove that the global $L$-function $L\left(s, \pi \times \pi^{\prime} \times \pi^{\prime \prime}\right)$ is entire unless all three representations are monomial. The poles were then determined by Ikeda I]. They are again at $s=0,1$ and therefore $s(s-1) L\left(s, \pi \times \pi^{\prime} \times \pi^{\prime \prime}\right)$ is always entire.

In our setting $\mathbf{G}=\operatorname{Spin}(8)$ and $\mathbf{M}$ is generated by $\left\{\alpha_{1}, \alpha_{3}, \alpha_{4}\right\}$ with the middle root $\alpha_{2}$ eliminated. It is easy to see

$$
\mathbf{M}=\left(S L_{2} \times S L_{2} \times S L_{2}\right) \times G L_{1} /( \pm I),
$$

where $I=\left(I_{2}, I_{2}, I_{2}, 1\right)$. Let $\omega_{0}=\omega \omega^{\prime} \omega^{\prime \prime}$, where $\omega, \omega^{\prime}$, and $\omega^{\prime \prime}$ are the corresponding central characters. Choose irreducible constituents $\pi_{0}, \pi_{0}^{\prime}, \pi_{0}^{\prime \prime}$ of $\pi \mid S L_{2}\left(\mathbb{A}_{F}\right)$, $\pi^{\prime}\left|S L_{2}\left(\mathbb{A}_{F}\right), \pi^{\prime \prime}\right| S L_{2}\left(\mathbb{A}_{F}\right)$, arbitrarily, and consider them as representations of three $S L_{2}$ components of $\mathbf{M}$. Finally, consider $\omega_{0}$ as a character of $G L_{1}\left(\mathbb{A}_{F}\right)$. This then leads to a representation $\Pi$ of $M=\mathbf{M}\left(\mathbb{A}_{F}\right)$. We have $m=2$,

$$
L\left(s, \Pi, r_{1}\right)=L\left(s, \pi \times \pi^{\prime} \times \pi^{\prime \prime}\right),
$$

and

$$
L\left(s, \Pi, r_{2}\right)=L\left(s, \omega_{0}\right) .
$$

Moreover, if $L\left(s, \pi \times \pi^{\prime} \times \pi^{\prime \prime}\right)$ is not entire, then $P_{1}(s)=s(s-1)$. We have:

Corollary 4.4. Suppose at least one of $\pi, \pi^{\prime}$, or $\pi^{\prime \prime}$ is non-monomial. Then the $L$-function $L\left(s, \pi \times \pi^{\prime} \times \pi^{\prime \prime}\right)$ is bounded in every vertical strip of finite width. The same is true in every $T_{\varepsilon, I}$, otherwise.

Proof. Assumption 2.1 is Proposition 3.2 of KSh2 in this case.

Remark 1. This was first proved by Ramakrishnan using the Rankin-Selberg method in Ra (Section 3.4). As explained there, this is absolutely necessary in order to prove the functorial lift corresponding to the $L$-group embedding

$$
G L_{2}(\mathbb{C}) \otimes G L_{2}(\mathbb{C}) \longrightarrow G L_{4}(\mathbb{C}),
$$

using the converse theorem, which was established by Ramakrishnan in [Ra] as well. This is a very important result with many fundamental applications such as multiplicity one for $S L_{2}\left(\mathbb{A}_{F}\right)$ (Section 4.1 of $[\mathrm{Ra}]$ ).

Remark 2. Ramakrishnan's proof is more specialized and complicated since he needs to deal with residual (non-cuspidal) Eisenstein series as opposed to ours. In spirit, our two proofs are close to each other (cf. Section 6).

Our final example is that of the Rankin-Selberg $L$-functions $L(s, \sigma \times \tau)$, where $\sigma$ is a cuspidal representation of $G L_{n}\left(\mathbb{A}_{F}\right)$ and $\tau$ is a generic cuspidal representation of $G_{p}, G_{p}=\mathbf{G}_{p}\left(\mathbb{A}_{F}\right)$, where $\mathbf{G}_{p}$ is a split classical group of rank $p$, i.e. either a split special orthogonal group or a symplectic one. The cases of similitude groups must be similar. But we have not checked that carefully. 
Suppose $v$ is such that $\sigma_{v}$ and $\tau_{v}$ are both unramified. Let $A_{v}$ and $A_{v}^{\prime}$ be the corresponding conjugacy classes, respectively. The local Langlands $L$-function is then simply

$$
L\left(s, \sigma_{v} \otimes \tau_{v}\right)=\operatorname{det}\left(I-A_{v} \otimes A_{v}^{\prime} q_{v}^{-s}\right)^{-1} .
$$

An enormous amount of work has been done about these $L$-functions (GPSR, So1, So2, GRS1, GRS2, [Sh1) and local factors are defined by both methods. Their equality can probably be verified using local-global methods along the lines of [Sh1], GRS2, and [Ra] (cf. Proposition 6.3 of GRS2]). But nothing as strong as the earlier cases can be said about these.

In our setting $\mathbf{G}=\mathbf{G}_{p+n}, \mathbf{M}=G L_{n} \times \mathbf{G}_{p}$, and $\pi=\sigma \otimes \tilde{\tau}$. Moreover, if $p>0$, then $m=2$ and

$$
L\left(s, \pi, r_{1}\right)=L(s, \sigma \times \tau) .
$$

The representation $r_{2}=\Lambda^{2} \rho_{n}$, or $\operatorname{Sym}^{2}\left(\rho_{n}\right)$, according as $\mathbf{G}$ is a symplectic or a special even orthogonal group, or a special odd orthogonal one. Here $\rho_{n}$ is the standard representation of $G L_{n}(\mathbb{C})$ and ${ }^{L} G_{p}$ acts trivially; in other words, according as ${ }^{L} G^{0}$ is special orthogonal or symplectic. Then

$$
L\left(s, \pi, r_{2}\right)=L\left(s, \sigma, r_{2}\right) .
$$

When $\mathbf{G}=S O_{2 p+2 n+1}$ and $\sigma$ satisfies the weak Ramanujan property (cf. [CPS2] and Definition 3.1 of [K2] $)$, then Kim ([K2], Theorem 3.5) has proved that $L(s, \sigma \times \tau)$ is holomorphic except possibly at $s=0,1 / 2,1$. In general, some rather weaker results are also proved in $\underline{\mathrm{K} 2}$ to cover the symplectic groups (Theorem 3.7 of [K2]). On the other hand, Assumption 2.1 is verified for all of these groups (Proposition 3.4 of [K2], Lemma 3.3 and Proposition 3.4 of [K1]). (The case of the even special orthogonal group is not proved there. But in view of Muic's new result [M2], it is totally similar.) Let $P_{1}(s)$ and $P_{2}(s)$ be the polynomials defined by the finite sets of poles of $L(s, \sigma \times \tau)$ and $L\left(s, \sigma, r_{2}\right), r_{2}=\Lambda^{2} \rho_{n}$ or $\operatorname{Sym}^{2}\left(\rho_{n}\right)$ (Proposition 3.2). Then

$$
P_{1}(s) L(s, \sigma \times \tau)
$$

and

$$
P_{2}(s) L\left(s, \sigma, r_{2}\right)
$$

are entire.

We have:

Corollary 4.5. Let $\sigma$ and $\tau$ be cuspidal representations of $G L_{n}\left(\mathbb{A}_{F}\right)$ and $G_{p}$. Assume $\tau$ is globally generic.

a) The $L$-function $L(s, \sigma \times \tau)$ has at most a finite number of poles in $\mathbb{C}$ and is bounded in vertical strips of finite width.

b) The same is true for $L\left(s, \sigma, \Lambda^{2} \rho_{n}\right)$ and $L\left(s, \sigma, \operatorname{Sym}^{2}\left(\rho_{n}\right)\right)$.

Remark 1. The $L$-functions $L(s, \sigma \times \tau)$ are quite important if one tries to use the converse theorem to lift cusp forms from classical groups to $G L_{r}$. This is a long-term project which has been studied by a number of experts and at present is being pursued by a subset of them, notably Cogdell and Piatetski-Shapiro. We observe that to apply the converse theorem a result such as that of Corollary $4.5 \mathrm{a}$ ) is absolutely necessary ([CPS1, CPS3], CPS4, Rag1, Rag2, Ra ). (The existence of a lifting from $S O_{2 n+1}$ to $G L_{2 n}$ is now proved in [CKPSS.) 
Remark 2. We expect the situation of quasisplit classical groups as well as similitudes of all these groups to follow the same path. But one first needs to verify Assumption 2.1 for them.

\section{Proof of Theorem 4.1}

With no loss of generality we may assume that $\pi$ is a representation of $M^{1}$ so that the poles are all real. Given a real number $\varepsilon>0$ and a real closed finite interval $I$, let $T_{\varepsilon, I}$ be as in Section 4. Suppose $I=[a, b]$ and $c$ is a real number. We will say $I \geq c$ if and only if $a \geq c$.

A complex function $f(s), s=\sigma+i t$, is said to be of finite order in $T_{\varepsilon, I}$ if it is analytic in $T_{\varepsilon, I}$, and there exist real numbers $K>0$ and $\rho>0$ such that

$$
|f(\sigma+i t)| \leq K e^{|t|^{\rho}}
$$

for all $s=\sigma+i t \in T_{\varepsilon, I}$. The smallest such $\rho$ is called the order of $f$. But in what follows we do not need the exact order of a function.

A meromorphic function $f(s)$ is said to be of finite order in a closed (open) half plane $\operatorname{Re}(s)=\sigma \geq \sigma_{0}\left(\sigma>\sigma_{0}\right.$, resp.) if it has only a finite number of poles there, and if the monic polynomial $P(s)$ gives the poles of $f(s)$ in $\sigma \geq \sigma_{0}\left(\sigma>\sigma_{0}\right.$, resp.), with multiplicities counted, then there exist real numbers $K>0$ and $\rho>0$, both depending only on $f$ and $\sigma_{0}$, such that

$$
|P(s) f(s)| \leq K e^{|s|^{\rho}}
$$

for all $\sigma \geq \sigma_{0}\left(\sigma>\sigma_{0}\right.$, resp.), $s=\sigma+i t$. We will use the same definition when dealing with finite strips (as opposed to half strips $T_{\epsilon, I}$ defined at the beginning of Section 4) or the whole complex plane.

The strategy of the proof is as follows. First, using some rather deep results in complex analysis (Matsaev Theorem; cf. Lecture 26 of [Le]) and the functional equation, we show that each $L\left(s, \pi, r_{i}\right)$ is of finite order in every $T_{\varepsilon, I}$ (in fact in the whole complex plane in the sense of our definition). Next we take a vertical strip $T_{\varepsilon, I}$ with $a \leq \operatorname{Re}(s) \leq b$ such that $L\left(s, \pi, r_{i}\right)$ is bounded on $\operatorname{Re}(s)=a$ and $\operatorname{Re}(s)=b$, for example by taking $b$ larger than an abscissa of absolute convergence and $a<-b$. The theorem is now a consequence of Phragmén-Lindelöf's theorem applied to such $T_{\varepsilon, I}$ (see for example $\S 5.6$ of $\mathrm{T}$, especially 5.61 and the first paragraph in 5.65). Thus the bulk of the proof falls on proving the finiteness of order for each $L\left(s, \pi, r_{i}\right)$ in each $T_{\varepsilon, I}$.

We start with the following lemma.

Lemma 5.1. Under Assumption 2.1 for every $\left(G_{v}, M_{v}, \tilde{\pi}_{v}\right)$, the product

$$
\prod_{i=1}^{m} L\left(i s, \pi, r_{i}\right) / L\left(1+i s, \pi, r_{i}\right)
$$

is of finite order in the closed half plane $\operatorname{Re}(s) \geq 1 / 2$.

Proof. We start with equation (3.3.1) applied to $\tilde{\pi}$. For simplicity of notation let

$$
\ell(s, \pi)=\prod_{i=1}^{m} \varepsilon\left(i s, \pi, r_{i}\right)^{-1} L\left(i s, \pi, r_{i}\right) / L\left(1+i s, \pi, r_{i}\right) .
$$

Evaluate both sides of (3.3.1) at the identity. Next let $u=\otimes_{v} u_{v}$ be a $K$-finite vector in the space of $\pi=\otimes_{v} \pi_{v}$ and consider the matrix coefficient of both sides 
of (3.3.1) at $u=\otimes_{v} u_{v}$. We then have

$$
\langle M(s, \tilde{\pi}) f(e), u\rangle=\ell(s, \pi) \prod_{v}\left\langle N\left(s \tilde{\alpha}, \tilde{\pi}_{v}, w\right) f_{v}(e), u_{v}\right\rangle .
$$

In the notation of $[\mathrm{HC}$ (cf. Theorem 5 of $[\underline{\mathrm{HC}}$, as well as [L2]), we may choose $f$ such that

$$
(M(s, \tilde{\pi}) f)(e)=c(w, s \tilde{\alpha}) \varphi
$$

for some $\varphi$ in the space of $\tilde{\pi}$. Then by the Schwarz Lemma, the absolute value of the left hand side of (5.1.3) is bounded by

$$
|c(w, s \tilde{\alpha})|\|\varphi\|\|u\|,
$$

where $|c(w, s \tilde{\alpha})|$ is the norm of $c(w, s \tilde{\alpha})$, and $\|\varphi\|$ and $\|u\|$ are the $L^{2}$-norms of $\varphi$ and $u$ in $\tilde{\pi}$ and $\pi$, respectively.

It follows from Lemma 101 of [HC] (also see page 242 of [L3]) that

$$
\ell(s, \pi) \prod_{v}\left\langle N\left(s \tilde{\alpha}, \tilde{\pi}_{v}, w\right) f_{v}(e), u_{v}\right\rangle
$$

is bounded in every $T_{\varepsilon, I}$ with $I \geq 0$. Combining this with Lemma 38 of $\underline{\mathrm{HC}}$ and the finiteness of poles of $c(w, s \tilde{\alpha}), \sigma \geq 0$, we see that (5.1.4) is in fact of finite order in the closed half plane $\sigma \geq 0$.

On the other hand, by part R6 of Theorem 2.1 of [A1] when $v$ is archimedean, as well as the results of [Si] and [Sh1] otherwise, each

$$
\left\langle N\left(s \tilde{\alpha}, \tilde{\pi}_{v}, w\right) f_{v}(e), u_{v}\right\rangle
$$

is a rational function of either $s$ if $v \mid \infty$, or $q_{v}^{-s}$ if $v$ is finite. Moreover, (5.1.5) is equal to 1 for almost all $v$.

Applying Assumption 2.1 for $\left(G_{v}, M_{v}, \tilde{\pi}_{v}\right)$ to $(5.1 .3)$, it is clear that $\ell(s, \pi)$ is analytic, except possibly for a finite number of poles coming from those of $M(s, \tilde{\pi})$. Observe that the $f_{v}$ used in applying Assumption 2.1 need not be among the components of our $f$ (and $\varphi$ ) which we have fixed throughout the proof.

Write $T(s)$ for the numerator of $\Pi_{v}\left\langle N\left(s \tilde{\alpha}, \tilde{\pi}_{v}, w\right) f_{v}(e), u_{v}\right\rangle$. Observe that the zeros of $T(s)$ will all lie discretely on a finite number of lines parallel to the imaginary axis. Therefore there exists a real number $\delta<1 / 2$ such that all the zeros of $T(s)$ with $\operatorname{Re}(s) \geq \delta$ are among those of $\langle M(s, \tilde{\pi}) f(e), u\rangle$ and consequently $\ell(s, \pi)$ is in fact analytic (except possibly for a finite number of poles) for $\operatorname{Re}(s)>\delta$. Since $\langle M(s, \tilde{\pi}) f(e), u\rangle$ and $\Pi_{v}\left\langle N\left(s \tilde{\alpha}, \tilde{\pi}_{v}, w\right) f_{v}(e), u_{v}\right\rangle$ are both of finite order in the same half plane, a standard division theorem (cf. part a) of our Theorem 5.7) implies the finiteness of the order of $\ell(s, \pi)$ for $\operatorname{Re}(s) \geq 1 / 2>\delta$. The lemma is now proved.

Next, let $E_{\chi}(s, \tilde{\varphi}, e, P)$ be the $\chi$-Fourier coefficient of the Eisenstein series attached to $\pi$ and $P$ as defined in [Sh2], Sh4]. Here $\tilde{\varphi}$ is the function attached to $f$ coming from a cusp form $\varphi$ on $M^{1}$. We record equation (3.4) of [Sh2] as:

Proposition 5.2. One has

$$
E_{\chi}(s, \tilde{\varphi}, e, P)=\prod_{v \in S} W_{v, s}(e) \cdot \prod_{i=1}^{m} L_{S}\left(1+i s, \pi, \tilde{r}_{i}\right)^{-1},
$$

where $W_{v, s}$ is the Whittaker function attached to $f_{v}$ by equation (3.2) of [Sh2].

Notice that we use the subscript $s$ to emphasize its dependence on $s \in \mathbb{C}$. We need: 
Lemma 5.3. a) Suppose $v$ is finite. Then each $f_{v}, v \in S$, can be chosen so that $W_{v, s}(e)$ becomes a non-zero constant (independent of $s$ ).

b) Assume $v$ is archimedean. Given $s \in \mathbb{C}, f_{v}^{0}$, a $K_{v}$-finite function in $V\left(0, \pi_{v}\right)$ defining $f_{v} \in V\left(s, \pi_{v}\right)$ by $f_{v}(g)=\exp \left\langle s \tilde{\alpha}, H_{P_{v}}(g)\right\rangle f_{v}^{0}(g)$, can be chosen so that $W_{v, s}(e) \neq 0$. This choice of $f_{v}^{0}$ need not be the same as the one for which Assumption 2.1 is supposed to be valid.

Proof. Part a) is well known. Thus assume $v$ is archimedean. The non-vanishing of $W_{v, s}(e)$ for some $C^{\infty}$-function $f_{v}$ in the space $V\left(s, \pi_{v}\right)$ is trivial (cf. [Sh3]). The $K$-finite case is then a consequence of continuity of the Whittaker functional $f_{v} \mapsto W_{s, v}(e)$ with respect to $C^{\infty}$-topology in $V\left(s, \pi_{v}\right)_{\infty}$ and the density of $K-$ finite vectors inside the smooth ones. (In the notation of [Sh3], this is the functional $\kappa(\nu, \sigma)$ with $\nu=s \tilde{\alpha}$ and $\sigma=\pi_{v}$. The result we are quoting is the corollary to Lemma 2.5 of [Sh3].)

Next we state the following important proposition to whose proof our next section is devoted.

Proposition 5.4. The Fourier coefficient $E_{\chi}(s, \tilde{\varphi}, e, P)$ of $E(s, \tilde{\varphi}, e, P)$ is a ratio of two holomorphic functions of finite order in every $T_{\varepsilon, I}$ with $I \geq 0$.

Using the estimate in Lemma 23 and Lemma 5 a of [HC] (with $\Omega=\{e\}$ ), we see that $E_{\chi}(s, \tilde{\varphi}, e, p)$ is holomorphic and of finite order for $\operatorname{Re}(s)$ sufficiently large.

Let $R(s)$ be the monic polynomial whose zeros are poles of

$$
\tilde{E}_{\chi}(s)=\prod_{v \in S} \prod_{i=1}^{m} L\left(1+i s, \pi_{v}, \tilde{r}_{i, v}\right)^{-1} E_{\chi}(s, \tilde{\varphi}, e, P)
$$

for $\operatorname{Re}(s) \geq 0$. Then by Lemma 5.3 and Proposition 5.2 both $R(s) \tilde{E}_{\chi}(s)$ and $R(s) \prod_{i=1}^{m} L\left(1+i s, \pi, \tilde{r}_{i}\right)^{-1}$ are holomorphic for $\operatorname{Re}(s) \geq 0$. It therefore follows that:

Lemma 5.5. The analytic function $R(s) \tilde{E}_{\chi}(s)$ is a ratio of two analytic functions of finite order in the closed half plane $\sigma \geq 0$.

Next we need to address the growth of archimedean Whittaker functionals $W_{v, s}(e)$ for each $v$.

Lemma 5.6. Fix $v=\infty$. The function $W_{v, s}(e)$ is entire and of finite order in the closed half plane $\operatorname{Re}(s) \geq 0$.

Proof. The first statement is well known (cf. [Sh3], [Sh9], GW]). For simplicity we will drop the index $v$; then $f$ will denote $f_{v}, f^{0}=f_{v}^{0}$, and $W_{s}=W_{v, s}$. Using Casselman's subrepresentation theorem $\pi \hookrightarrow I_{M}\left(\nu_{0}, \eta_{0}\right)$, a principal series representation of $M$, with $\operatorname{Re}\left(\nu_{0}\left(H_{\beta}\right)\right) \geq 0, \beta \in \theta, \mathbf{M}=\mathbf{M}_{\theta}$ (cf. [Sh3]). Then $I(s \tilde{\alpha}, \pi) \hookrightarrow I\left(\tilde{\nu}, \eta_{0}\right), \pi=\pi_{v}$, where $\tilde{\nu}=s \tilde{\alpha}+\nu_{0}$. Let $\tilde{f}$ be the image of $f$ under this embedding. Note that $\operatorname{Re}\left(\tilde{\nu}\left(H_{\beta}\right)\right) \geq 0, \beta \in \theta$. But $\operatorname{Re}\left(\tilde{\nu}\left(H_{\alpha}\right)\right)$ may be negative, even if $\operatorname{Re}(s) \geq 0$. If $\tilde{\nu}$ is in the positive Weyl chamber, then $W_{v, s}(e)$, which is now a value of the Jacquet integral for $I\left(\tilde{\nu}, \eta_{0}\right)$, is bounded by

$$
\left|W_{s}(e)\right| \leq\left|C_{0}(s)\right|\|\tilde{f}\|,
$$

where $\|\tilde{f}\|=\sup _{k \in K}|\tilde{f}(k)|, K=K_{v}$. Here $C_{0}(s)$ is a product of ratios of $\Gamma$-functions, each ratio given explicitly by equation (2.2.5) of [Sch] (cf. equation (2.2), page 304 
of [Sh4] and Theorem 5.4 of [GW]). By the inequality on $\Gamma$-functions given in page 37 of A1] it is bounded by a rational function for $\operatorname{Re}(s) \geq 0$, since each $\lambda_{\beta}$ in equation (2.2.5) of [Sch] now has a positive real part.

On the other hand, suppose $\tilde{\nu}$ is not in the closure of the positive Weyl chamber. This will only happen if $\operatorname{Re}(s) \leq \sigma_{0}$ for some $\sigma_{0}$ depending only on $\pi=\pi_{v}$. To complete the lemma we only need to prove it in the strip $0 \leq \operatorname{Re}(s) \leq \sigma_{0}$. We use the functional equation for Whittaker functions. Let $A(\tilde{\nu}, \omega)$ be a standard intertwining operator for which $w$ is of shortest length and $w \tilde{\nu}$ is in the closure of the positive Weyl chamber. Thus $A(\tilde{\nu}, w)$ sends $I\left(\tilde{\nu}, \eta_{0}\right)$ into $I\left(w \tilde{\nu}, w \eta_{0}\right)$. Let $C_{\chi}(\tilde{\nu}, w)$ be the corresponding local coefficient, i.e. the complex function $C_{\chi}(\tilde{\nu}, w)$ defined by

$$
W_{s}(e)=C_{\chi}(\tilde{\nu}, w) \lambda(A(\tilde{\nu}, w) \tilde{f}),
$$

where $\lambda$ is the canonical Whittaker functional for $I\left(w \tilde{\nu}, w \eta_{0}\right)$ (cf. [Sh3] and [Sh4]).

We will prove the finiteness of order in the strip $0 \leq \operatorname{Re}(s) \leq \sigma_{0}$ by induction on the length of $w$. Suppose for a simple reflection $w_{\gamma}, \ell\left(w w_{\gamma}^{-1}\right)<\ell(w)$, where $\ell(w)$ denotes the length of $w$. Then

$$
\left|W_{s}(e)\right|=\left|C_{\chi}\left(\tilde{\nu}, w_{\gamma}\right)\right|\left|\lambda\left(A\left(\tilde{\nu}, w_{\gamma}\right) \tilde{f}\right)\right| .
$$

Using the explicit formulas for the rank one local coefficients $C_{\chi}\left(\tilde{\nu}, w_{\gamma}\right)$ given in [Sh3] and [GW] and properties of $\Gamma$-functions, one immediately sees that $C_{\chi}\left(\tilde{\nu}, w_{\gamma}\right)$ is of finite order in the closed interval $0 \leq \operatorname{Re}(s) \leq \sigma_{0}$, having only a finite number of poles there. Our induction hypothesis is now that: There exist seminorms $\mu_{j}$ on $I\left(\tilde{\nu}, \eta_{0}\right)_{\infty}$ of the form

$$
\mu_{j}(\tilde{f})=\sup _{k \in K}\left|X_{j} \tilde{f}(k)\right|,
$$

each for some $X_{j}$ in the universal enveloping algebra of the complexified Lie algebra of $G=G_{v}$; and complex functions $\omega_{j}$ of finite order in the strip $0 \leq \operatorname{Re}(s) \leq \sigma_{0}$ such that

$$
\left|W_{s}(e)\right| \leq \sum_{j}\left|\omega_{j}(s)\right| \mu_{j}(\tilde{f})
$$

Clearly (5.6.1) implies the validity of the first step of induction. Now by the induction hypothesis there exist seminorms $\mu_{\ell}^{\prime}$ on $I\left(w_{\gamma}(\tilde{\nu}), w_{\gamma}\left(\eta_{0}\right)\right)_{\infty}$ and functions $\omega_{\ell}^{\prime}$ of finite order in $0 \leq \operatorname{Re}(s) \leq \sigma_{0}$ such that

$$
\left|\lambda\left(A\left(\tilde{\nu}, w_{\gamma}\right) \tilde{f}\right)\right| \leq \sum_{\ell}\left|\omega_{\ell}^{\prime}(s)\right| \mu_{\ell}^{\prime}\left(A\left(\tilde{\nu}, w_{\gamma}\right) \tilde{f}\right) .
$$

Moreover

$$
\begin{aligned}
\mu_{\ell}^{\prime}\left(A\left(\tilde{\nu}, w_{\gamma}\right) \tilde{f}\right) & =\sup _{k \in K}\left|X_{\ell}^{\prime}\left(A\left(\tilde{\nu}, w_{\gamma}\right) \tilde{f}\right)\right| \\
& =\sup _{k \in K}\left|A\left(\tilde{\nu}, w_{\gamma}\right)\left(X_{\ell}^{\prime} \tilde{f}\right)\right| .
\end{aligned}
$$

We now appeal to Schiffmann's estimates (2.3.12) and (2.3.14) for rank one intertwining operators in page 40 of $[\underline{\mathrm{Sch}}]$ (applied to $\Omega^{\prime}=\{\tilde{\nu}\}$ and $\Omega=\{e\}$, also cf. estimate (7.38) of [GW]) to conclude

$$
\mu_{\ell}^{\prime}\left(A\left(\tilde{\nu}, w_{\gamma}\right) \tilde{f}\right) \leq \sum_{p}\left|\omega_{p}^{\prime \prime}\left(\tilde{\nu}\left(H_{\gamma}\right)\right)\right| \mu_{p}^{\prime \prime}(\tilde{f})
$$


with seminorms $\mu_{p}^{\prime \prime}$ of the above type on $I\left(\tilde{\nu}, \eta_{0}\right)$, where functions $\omega_{p}^{\prime \prime}$ are of finite order in $\operatorname{Re}(s) \geq 0$.

Observe that $\tilde{f}$ depends on $\tilde{\nu}$ and therefore $s$, and consequently the same is true of $\left\{\mu_{p}^{\prime \prime}(\tilde{f})\right\}$. To take this into account, we appeal to [GW]. We use notation as in GW]. By Lemma 5.1 of [GW] there exist positive constants $a, b$, and $M$ such that

$$
\|\tilde{f}\|_{\mathcal{Z}_{\tilde{\nu}}, 1, a|\tilde{\nu}|+b} \leq M\|\tilde{f}\|_{\mathcal{Y}, 1,1}
$$

where $\mathcal{Z}_{\tilde{\nu}}=\left\{I\left(\tilde{\nu}, \eta_{0}\right)\left(Z_{i}\right)\right\}$ and $\mathcal{Y}=\left\{I\left(0, \eta_{0}\right)\left(E_{j}\right)\right\}$. Here $\left\{Z_{i}\right\}$ and $\left\{E_{j}\right\}$ are fixed bases for complex Lie algebras of $G$ and $K$, respectively, and

$$
\|\tilde{f}\|_{\mathcal{Y}, 1,1}=\max _{j}\left\{\sup _{k \in K}\left|E_{j} \tilde{f}^{0}(k)\right|\right\}
$$

(cf. Theorem 1.1 and definitions in pages 208 and 209 of [GW]), which is obviously independent of $\tilde{\nu}$.

But for each $i$, definition (1.2) in page 208 of [GW] with $n=\lambda=1$ implies

$$
\left\|Z_{i} \tilde{f}\right\| \leq(a|\tilde{\nu}|+b)\|\tilde{f}\|_{\mathcal{Z}_{\tilde{\nu}, 1, a|\tilde{\nu}|+b}}
$$

We conclude that given $X$ in the universal enveloping algebra, there exists a positive integer $N$ such that

$$
\begin{aligned}
\sup _{k \in K}|X \tilde{f}(k)| & =\|X \tilde{f}\| \\
& \leq M(a|\tilde{\nu}|+b)^{N}\|\tilde{f}\|_{\mathcal{Y}, 1,1} .
\end{aligned}
$$

The induction hypothesis for $w$ is now proved. Since $W_{v, s}(e)$ is entire, it will be bounded on any compact set containing the poles of functions that appear in front of different $\|\tilde{f}\|_{\mathcal{Y}, 1,1}$ (attached to different $X$ 's) in the interval $0 \leq \operatorname{Re}(s) \leq \sigma_{0}$. The lemma now follows.

To continue we need the following theorem from complex analysis which was communicated to us by M. Sodin (cf. Lecture 26 of [Le] on Matsaev's Theorem). We are indebted to him for countless hours that he spent in helping us in resolving the division problem whose resolution is needed in order to handle the quotients that appear in different expressions for $L$-functions.

Theorem 5.7 (Matsaev, lecture 26 of [Le]). Let $f(z)$ and $h(z)$ be analytic functions in the closed right half plane $x \geq 0, z=x+i y$, satisfying

$$
\log |f(z)| \leq C\left(|z|^{\rho}+1\right)
$$

and

$$
\log |h(z)| \leq C\left(|z|^{\rho}+1\right)
$$

for positive numerical constants $C$ and $\rho>1$. Let $g(z)=f(z) / h(z)$.

a) Assume $g(z)$ is analytic for $x \geq 0$. Then it is of (finite) order less than or equal to $\rho+1$ in any vertical strip $T_{I}, I>0$.

b) Suppose further that $g(z)$ is entire and there exist constants $\alpha \in \mathbb{R}$ and $C_{1}>0$ such that

$$
|g(-x+i y)|=C_{1} e^{\alpha x}|g(x+i y)|
$$

for any $x>0$. Then $g(z)$ is of (finite) order less than or equal to $\rho$ in all of $\mathbb{C}$. 
Proof. a) Since $g=f / h$ is analytic, we can majorize $\log |g(z)|$ by the Poisson integral in each semi-disk $\{|z|<R, \operatorname{Re}(z)>0\}$ (cf. equation 14, page 193 of [Le]):

$$
\begin{aligned}
\log |g(z)| & \leq \int_{-\pi / 2}^{\pi / 2} K_{1}\left(\operatorname{Re}^{i \varphi}, z\right) \log \left|g\left(\operatorname{Re}^{i \varphi}\right)\right| d \varphi \\
& +\int_{-R}^{R} K_{2}(i \tau, z) \log |g(i \tau)| d \tau,
\end{aligned}
$$

where

$$
K_{1}\left(\operatorname{Re}^{i \varphi}, z\right)=\frac{R^{2}-|z|^{2}}{2 \pi}\left(\frac{1}{\left|\operatorname{Re}^{i \varphi}-z\right|^{2}}-\frac{1}{\left|\operatorname{Re}^{i \varphi}-z^{*}\right|^{2}}\right),
$$

$z^{*}=-x+i y$, and

$$
K_{2}(i \tau, z)=\frac{x}{\pi}\left(\frac{1}{|i \tau-z|^{2}}-\frac{R^{2}}{\left|R^{2}+i \tau z\right|^{2}}\right)
$$

Using the positivity of Poisson kernels Le, we can replace $\log |g|$ by $\log ^{+}|g|$ under the integral sign, to conclude

$$
\begin{aligned}
\log |g(z)| & \leq \int_{-\pi / 2}^{\pi / 2} K_{1}\left(\operatorname{Re}^{i \varphi}, z\right) \log ^{+}\left|g\left(\operatorname{Re}^{i \varphi}\right)\right| d \varphi \\
& +\int_{-R}^{R} K_{2}(i \tau, z) \log ^{+}|g(i \tau)| d \tau .
\end{aligned}
$$

Next, assuming $r=|z|=R / 2, r \geq 2, \theta=\arg (z)$, we estimate the kernels $K_{1}$ and $K_{2}$ as follows: for $K_{1}$ we have

$$
K_{1}\left(\operatorname{Re}^{i \varphi}, z\right) \leq \frac{R^{2}-r^{2}}{2 \pi} \cdot \frac{4 r R \cos \theta \cos \varphi}{(R-r)^{4}} \leq \frac{12 \cos \varphi}{\pi}
$$

(cf. equation (12), page 211 of [Le for details); since

$$
|i \tau-z|^{2} \geq \begin{cases}\tau^{2} \cos ^{2} \theta, & |\tau| \geq 1 \\ r^{2} / 4, & |\tau|<1\end{cases}
$$

we obtain

$$
\begin{aligned}
K_{2}(i \tau, z) & \leq \frac{R \cos \theta}{2 \pi} \cdot \begin{cases}\frac{1}{\tau^{2} \cos ^{2} \theta}, & |\tau| \geq 1, \\
\frac{4}{r^{2}}, & |\tau|<1,\end{cases} \\
& = \begin{cases}\frac{R}{2 \pi \tau^{2} \cos \theta}, & |\tau| \geq 1, \\
\frac{8 \cos \theta}{\pi R}, & |\tau|<1\end{cases}
\end{aligned}
$$

(cf. page 211 of [Le] again). Now, plugging estimates (5.7.5) and (5.7.6) in (5.7.4), we get

$$
\begin{aligned}
\log |g(z)| & \leq \frac{12}{\pi} \int_{-\pi / 2}^{\pi / 2} \log ^{+}\left|g\left(\operatorname{Re}^{i \varphi}\right)\right| \cos \varphi d \varphi \\
& +\frac{R}{2 \pi \cos \theta} \int_{1}^{R}\left(\log ^{+}|g(i \tau)|+\log ^{+}|g(-i \tau)|\right) \cdot d \tau / \tau^{2} \\
& +O(1)
\end{aligned}
$$

as $R \rightarrow \infty$. 
We now appeal to Carleman's formula (cf. [Le]) to estimate the right hand side of (5.7.7). Observe that

$$
\log ^{+}|g| \leq \log ^{+}|f|+\log ^{-}|h|,
$$

and consequently

$$
\begin{aligned}
& \frac{1}{\pi R} \int_{-\pi / 2}^{\pi / 2} \log ^{+}\left|g\left(\operatorname{Re}^{i \varphi}\right)\right| \cos \varphi d \varphi \\
& \quad+\frac{1}{2 \pi} \int_{1}^{R}\left(\log ^{+}|g(i \tau)|+\log ^{+}|g(-i \tau)|\right) d \tau / \tau^{2} \\
& \leq \frac{1}{\pi R} \int_{-\pi / 2}^{\pi / 2} \log ^{+}\left|f\left(\operatorname{Re}^{i \varphi}\right)\right| \cos \varphi d \varphi \\
& \quad+\frac{1}{2 \pi} \int_{1}^{R}\left(\log ^{+}|f(i \tau)|+\log ^{+}|f(-i \tau)|\right) \cdot d \tau / \tau^{2} \\
& \quad+\frac{1}{\pi R} \int_{-\pi / 2}^{\pi / 2} \log ^{-}\left|h\left(\operatorname{Re}^{i \varphi}\right)\right| \cos \varphi d \varphi \\
& \quad+\frac{1}{2 \pi} \int_{1}^{R}\left(\log ^{-}|h(i \tau)|+\log ^{-}|h(-i \tau)|\right) \cdot d \tau / \tau^{2} \\
& \quad+O(1),
\end{aligned}
$$

as $R \rightarrow \infty$. Applying Carleman's formula to $h$ we conclude that (5.7.8) is bounded above by

$$
\begin{aligned}
& \frac{1}{\pi R} \int_{-\pi / 2}^{\pi / 2}\left(\log ^{+}\left|f\left(\operatorname{Re}^{i \varphi}\right)\right|+\log ^{+}\left|h\left(\operatorname{Re}^{i \varphi}\right)\right|\right) \cos \varphi d \varphi \\
+ & \frac{1}{2 \pi} \int_{1}^{R}\left(\log ^{+}|f(i \tau)|+\log ^{+}|f(-i \tau)|+\log ^{+}|h(i \tau)|+\log ^{+}|h(-i \tau)|\right) \cdot d \tau / \tau^{2} \\
+ & O(1),
\end{aligned}
$$

as $R \rightarrow \infty$. Substituting this into (5.7.7) we obtain

$$
\begin{aligned}
& \log |g(z)| \leq \frac{D}{\cos \theta} \int_{-\pi / 2}^{\pi / 2}\left(\log ^{+}\left|f\left(\operatorname{Re}^{i \varphi}\right)\right|+\log ^{+}\left|h\left(\operatorname{Re}^{i \varphi}\right)\right|\right) \cos \varphi d \varphi \\
& +\int_{1}^{R}\left(\log ^{+}|f(i \tau)|+\log ^{+}|f(-i \tau)|+\log ^{+}|h(i \tau)|+\log ^{+}|h(-i \tau)|\right) \cdot d \tau / \tau^{2} \\
& +O(1)
\end{aligned}
$$

where $z=r e^{i \theta}, R=2 r$, and as $r \rightarrow \infty$, for some constant $D$. Substituting (5.7.1) and (5.7.2) into (5.7.9), one immediately gets a bound

$$
\log |g(z)| \leq C^{\prime}\left(|z|^{\rho+1}+1\right)
$$

using the inequality $(\cos \theta)^{-1}=r / a$, if the interval $I$ defining $T_{I}$ is given by $I=$ $[a, b], a>0$. This implies part a).

To prove part b) we again plug (5.7.1) and (5.7.2) into (5.7.8), but this time keeping $\cos \theta$. We get

$$
\log |g(z)| \leq \frac{C_{1}}{\cos \theta}\left(|z|^{\rho}+1\right)
$$


for $|\theta|<\pi / 2$. Now using functional equation (5.7.3), we extend the estimate (5.7.11) to all of $\mathbb{C}$ with $|\cos \theta|$ in the denominator and possibly a larger $C_{1}$. The statement about the order of $g(z)$ in part b) of the theorem is now a consequence of Theorem 3 in lecture 26, page 212, of Le].

Remark 5.8. The conditions of Theorem 5.7 can be further relaxed, if needed. Instead of (5.7.1) and (5.7.2), it is enough to have an upper bound of the form

$$
\frac{C}{x^{\ell}}\left(|z|^{\rho}+1\right) \quad(\ell>0, x>0) .
$$

A more general functional equation of the form

$$
|P(z)||g(-x+i y)|=|Q(z)||g(x+i y)|
$$

in which $P$ and $Q$ are entire functions of order less than or equal to $\rho$, can also replace (5.7.3).

Proof of Theorem 4.1. We are now ready to prove Theorem 4.1. We continue to assume Assumption 2.1 in everything that follows. Let us now state our induction hypothesis:

Induction Hypothesis. For each quasisplit connected reductive group over $F$ and every maximal parabolic subgroup $\mathbf{P}=\mathbf{M N}$, let $r=\bigoplus_{i=1}^{m} r_{i}$ be the adjoint action of ${ }^{L} M$ on ${ }^{L} \mathfrak{n}$. Then for $i \geq 2$, every $L\left(s, \pi, r_{i}\right)$ is of finite order in the whole complex plane (in our sense, i.e. allowing a finite number of poles in $\mathbb{C}$; cf. Proposition 3.2).

We will prove Theorem 4.1 by induction on $m$. By Lemma 5.1 and the functional equation, the first step of induction, i.e. $m=1$, is clear. We thus assume that the statement of the hypothesis is valid for all $m^{\prime}<m$. Let

$$
m(s, \pi)=\prod_{i=1}^{m} L\left(i s, \pi, r_{i}\right) / L\left(1+i s, \pi, r_{i}\right) .
$$

Moreover, for each $2 \leq i \leq m$, let $E_{\chi}^{i}(s)$ be the Fourier coefficient of the Eisenstein series $E^{i}$ attached to the parabolic subgroup $\mathbf{P}_{i}=\mathbf{M}_{i} \mathbf{N}_{i}$ of the group $\mathbf{G}_{i}$ in Proposition 3.1 on our general induction, which defines $L\left(s, \pi, r_{i}\right)$ as its first $L$-function $L\left(s, \pi^{\prime}, r_{1}^{\prime}\right)$. Let

$$
\tilde{E}_{\chi}^{i}(s)=\prod_{v \in S} \prod_{j=1}^{m^{\prime}} L\left(1+j s, \pi_{v}^{\prime}, r_{j}^{\prime}\right)^{-1} E_{\chi}^{i}(s)
$$

and denote by $W_{v, s}^{i}(e), v \mid \infty$, each archimedean Whittaker functional for our $E^{i}$. Fixing $W_{v, s}^{i}(e)=1$ for all $v \in S, v<\infty$, we can write equation (5.2.1) of Proposition 5.2 for each $E_{\chi}^{i}(s), 2 \leq i \leq m$, as

$$
L\left(1+s, \pi, r_{i}\right)^{-1}=\prod_{j=2}^{m^{\prime}} L\left(1+j s, \pi^{\prime}, r_{j}^{\prime}\right) \tilde{E}_{\chi}^{i}(s) / \prod_{v \mid \infty} W_{v, s}^{i}(e) .
$$

(We change our data to get $r_{i}$ rather than $\tilde{r}_{i}$.)

Next, it follows from (5.2) that

$$
L\left(s, \pi, r_{1}\right)=m(s, \pi) L\left(1+s, \pi, r_{1}\right) \prod_{i=2}^{m} L\left(1+i s, \pi, r_{i}\right) \cdot L\left(i s, \pi, r_{i}\right)^{-1} .
$$


We will now assume that $\operatorname{Re}(s) \geq 1 / 2$ in (5.4), and for each inverse $L$-function $L\left(i s, \pi, r_{i}\right)^{-1}$, substitute the right hand side of (5.3) for the appropriate value of $s$. More precisely, equation (5.3) together with Lemmas 5.5 and 5.6 applied to $\tilde{E}_{\chi}^{i}(s)$ and $W_{v, s}^{i}(e)$, respectively, and the induction hypothesis for each $L\left(1+j s, \pi^{\prime}, r_{j}^{\prime}\right)$, show the existence of a pair of complex functions $f_{i}(s)$ and $h_{i}(s)$, each of finite order in the closed half plane $\operatorname{Re}(s) \geq 0$ (in our sense of allowing a finite number of poles), such that

$$
L\left(1+s, \pi, r_{i}\right)^{-1}=f_{i}(s) / h_{i}(s) .
$$

Rewriting (5.4) as

$$
L\left(s, \pi, r_{1}\right) / L\left(1+s, \pi, r_{1}\right)=m(s, \pi) \prod_{i=2}^{m} L\left(1+i s, \pi, r_{i}\right) \prod_{i=2}^{m} f_{i}(i s-1) / h_{i}(i s-1)
$$

and appealing to Lemma 5.1, the induction hypothesis, and the stated properties of $f_{i}(s)$ and $h_{i}(s)$ for each $i, 2 \leq i \leq m$, we see that the right hand side of (5.6) can be written as the ratio of two functions $F(s)$ and $H(s)$ of finite order in $\operatorname{Re}(s) \geq 1 / 2$. Write

$$
L\left(s, \pi, r_{1}\right) / L\left(1+s, \pi, r_{1}\right)=F(s) / H(s) .
$$

We now start with a line of absolute convergence for $L\left(s, \pi, r_{1}\right)$ and using the finiteness of poles for it on all of $\mathbb{C}$, apply part a) of Theorem 5.7 to $L\left(s, \pi, r_{1}\right)$ for $\operatorname{Re}(s) \geq 3 / 2$, to conclude inductively from (5.7) that

$$
L\left(s, \pi, r_{1}\right)=\tilde{f}(s) / \tilde{h}(s),
$$

where both $\tilde{f}(s)$ and $\tilde{h}(s)$ are complex functions of finite order in $\operatorname{Re}(s) \geq 1 / 2$.

Finally, if $P_{\pi}(s)=P_{1}(s)$ is the monic polynomial giving the poles of $L\left(s, \pi, r_{1}\right)$ as in Section 4 , then

$$
\begin{aligned}
L_{0}\left(s, \pi, r_{1}\right) & =P_{\pi}(s) L\left(s, \pi, r_{1}\right) \\
& =f_{0}(s) / h_{0}(s)
\end{aligned}
$$

where $f_{0}(s), h_{0}(s)$, and $L_{0}\left(s, \pi, r_{1}\right)$ are analytic in the closed half plane $\operatorname{Re}(s) \geq 1 / 2$. Observe that, using the functional equation,

$$
P_{\tilde{\pi}}(1-s)=P_{\pi}(s)
$$

and therefore

$$
L_{0}\left(s, \pi, r_{1}\right)=\varepsilon\left(s, \pi, r_{1}\right) L_{0}\left(1-s, \pi, \tilde{r}_{1}\right) .
$$

The conclusion is that

$$
L_{0}\left(s, \pi, r_{1}\right)=f_{0}(s) / h_{0}(s)
$$

where $f_{0}(s)$ and $h_{0}(s)$ are analytic functions of finite order in the closed half plane $\operatorname{Re}(s) \geq 1 / 2$, while $L_{0}\left(s, \pi, r_{1}\right)$ is entire. We now change variables $s=z+1 / 2, z=$ $x+i y$, and set

$$
g(z)=L_{0}\left(z+1 / 2, \pi, r_{1}\right) .
$$


Then

$$
\begin{aligned}
L_{0}\left(\frac{1}{2}-z, \pi, \tilde{r}_{1}\right) & =\overline{L_{0}\left(\frac{1}{2}-\bar{z}, \pi, r_{1}\right)} \\
& =\overline{g(-\bar{z})} \\
& =\overline{g(-x+i y)} .
\end{aligned}
$$

The functional equation implies

$$
g(x+i y)=\varepsilon\left(s, \pi, r_{1}\right) \overline{g(-x+i y)}
$$

or

$$
|g(-x+i y)|=C_{1} e^{\alpha x}|g(x+i y)|
$$

for $x>0$, where $C_{1}>0$ and $\alpha \in \mathbb{R}$. Set $f(z)=f_{0}(z+1 / 2)$ and $h(z)=h_{0}(z+1 / 2)$, which are now analytic of finite order in $\operatorname{Re}(z) \geq 0$ and $g(z)=f(z) / h(z)$. We now apply part b) of Theorem 5.7 to conclude the finiteness of order for $g(z)$ on all of $\mathbb{C}$. Thus, granting Proposition 5.4, which we will prove next, Theorem 4.1 is now proved.

\section{Analytic Behaviour of Fourier coefficients of CUSPidal Eisenstein Series; Proof of Proposition 5.4}

In this section we complete the proof of Theorem 4.1 by proving Proposition 5.4. As before, we rely on Langlands' theory of Eisenstein series ([L2], [L3], [HC], [MW1]). Our main references are Arthur's truncation ([A2], [A3]), Langlands' inner product formula ([A3], [L2], [L3]), Harish-Chandra [HC], and Muller [Mu] (see our Introduction). In fact, our original proof relied on $[\mathrm{HC}$, in which the inner product formula and more generally the analytic continuation of the Eisenstein series were proved using the Maass-Selberg relation for a semisimple algebraic group over $\mathbb{Q}$. Since the Maass-Selberg relation was proved in [HC] with only sketches provided, we felt that it may attract criticism for the lack of a complete proof. For this reason, and due to our use of adelic language, we have decided to use results of Arthur and Langlands. We thank A. Borel for his insistence that we do this; indeed, it was Borel who first complained to us about the proof of Harish-Chandra's MaassSelberg relation, and wanted a better proof in the language of adeles.

Finally, we should point out that in the special case of a symplectic group of rank 3, the same kind of arguments are used by Ramakrishnan in $\mathrm{Ra}$ to establish the same result as our Proposition 5.4 but for a non-cuspidal Eisenstein series. We refer to Corollary 4.4 in our Section 4 to explain Ramakrishnan's results [Ra].

The quasisplit group $\mathbf{G}_{0}=\operatorname{Res}_{F / \mathbb{Q}} \mathbf{G}$ is defined over $\mathbb{Q}$ and $\mathbf{G}_{0}\left(\mathbb{A}_{Q}\right)=\mathbf{G}\left(\mathbb{A}_{F}\right)$. Therefore with no loss of generality we may and will assume that $\mathbf{G}$ is defined over $\mathbb{Q}$ so as to be able to use Arthur.

Let $E(g)=E(s, \tilde{\varphi}, g, P)$ be our Eisenstein series as in [HC], [Sh4], [L2]. Given any parabolic subgroup $\mathbf{P}_{1}=\mathbf{M}_{1} \mathbf{N}_{1}, \mathbf{P}_{1} \supset \mathbf{B}$, let $E_{P_{1}}$ be the corresponding constant term

$$
E_{P_{1}}(g)=\int_{\mathbf{N}_{1}(\mathbb{Q}) \backslash N_{1}} E(n g) d n .
$$

Next, let $T$ be a sufficiently regular (cf. [A2], [A3]) element of $\mathfrak{a}_{0}$, where $\mathfrak{a}_{0}$ is the real Lie algebra of $\mathbf{A}_{0}$. This means that $\beta(T)$ is sufficiently large for all $\beta \in \Delta$. 
Let $\Lambda^{T}$ be the truncation operator of Arthur ([A2], A3] $)$. Then, $\pi$ being cuspidal,

$$
\Lambda^{T} E(g)=E(g)-\sum_{\mathbf{P}_{1}} \sum_{\delta \in \mathbf{P}_{1}(\mathbb{Q}) \backslash \mathbf{G}(\mathbb{Q})} E_{P_{1}}(\delta g) \hat{\tau}_{\mathbf{P}_{1}}\left(H_{P_{1}}(\delta g)-T\right),
$$

$g \in G^{1}$, where the first sum runs over the set $\left\{\mathbf{P}, \mathbf{P}^{\prime}\right\}$, with $\mathbf{P}^{\prime}=\mathbf{M}^{\prime} \mathbf{N}^{\prime}, \mathbf{N}^{\prime} \subset$ $\mathbf{U}, \mathbf{M}^{\prime}=w^{-1} \mathbf{M} w, \mathbf{M}^{\prime} \supset \mathbf{T} \supset \mathbf{A}_{0}$. Observe that if $\mathbf{M}$ is self-conjugate, then $\mathbf{P}^{\prime}=\mathbf{P}$ and therefore the set $\left\{\mathbf{P}, \mathbf{P}^{\prime}\right\}$ consists of only one element. Let us recall how $\hat{\tau}_{\mathbf{P}_{1}}$ is defined (cf. page 916 of $\left[\mathrm{A} 2\right.$ ). Let $\alpha_{1}$ be the unique simple root of $\mathbf{A}_{1}$, the split component of $\mathbf{M}_{1}, \mathbf{P}_{1}=\mathbf{M}_{1} \mathbf{N}_{1}, \mathbf{N}_{1} \subset \mathbf{U}, \mathbf{M}_{1} \supset \mathbf{T} \supset \mathbf{A}_{0}$, in $\mathbf{N}_{1}$. Then there exists a unique $\beta_{1} \in \Delta$ such that $\alpha_{1}=\beta_{1} \mid \mathfrak{a}_{1}$. Let $\alpha_{1}^{\vee}$ be the projection onto $\mathfrak{a}_{1}$ of the coroot $\beta_{1}^{\vee} \in \mathfrak{a}_{0}$ attached to $\beta_{1}$, where $\mathfrak{a}_{0}$ is the real Lie algebra of $\mathbf{A}_{0}$. Let $\varpi_{\alpha_{1}} \in \mathfrak{a}_{1}^{*}$ be dual to $\alpha_{1}^{\vee}$. Then $\varpi_{\alpha_{1}} \in \mathfrak{a}_{0}^{*}$. Now $\hat{\tau}_{\mathbf{P}_{1}}$ is the characteristic function in $\mathfrak{a}_{0}$ of the set

$$
\left\{H \in \mathfrak{a}_{0} \mid \varpi_{\alpha_{1}}(H)>0\right\} .
$$

As in [MW1] one can simply define $\hat{\tau}_{\mathbf{P}}$ to be the characteristic function of the sum of $\mathfrak{z}$, the real Lie algebra of the center of $\mathbf{G}$, and the interior of the cone generated by $\alpha_{1}$. We then need to project $T$ on $\mathfrak{a}_{1}$.

From now on we use $E(s, x), x \in G^{1}$, to denote our Eisenstein series. Then (cf. [Sh4] and Section 5 here)

$$
\left|E_{\chi}(s, \tilde{\varphi}, e, P)\right| \leq \int_{\mathbf{U}(\mathbb{Q}) \backslash U}|E(s, u)| d u .
$$

Let $\Omega \subset G^{1}$ be a compact set such that $\mathbf{U}(\mathbb{Q}) \backslash U \simeq \Omega$. Then there exists a constant $c>0$ such that

$$
\int_{\mathbf{U}(\mathbb{Q}) \backslash U}|E(s, u)| d u \leq c \sup _{u \in \Omega}|E(s, u)| .
$$

Cover $\Omega$ by open neighborhoods chosen as in Lemma 3.4.7 of [Ra] and fix a finite set $\left\{V_{i}\right\}$ of such neighborhoods covering $\Omega$, once and for all. Then by Lemma 3.4.7 of [Ra], there exists a polynomial $\tilde{P}(s)$ such that

$$
\left|E_{\chi}(s, \tilde{\varphi}, e, P)\right| \leq|\tilde{P}(s)| \cdot \max _{i}\|E(s)\|_{2, V_{i}},
$$

where \|\|$_{2, V_{i}}$ is the $L^{2}$-norm on $V_{i}$. Since $\wedge^{T} E(s)=\wedge^{T} E(s, \cdot)$ is square integrable on $\mathbf{G}(\mathbb{Q}) \backslash G^{1}$,

$$
\|E(s)\|_{2, V_{i}}^{2} \leq\left\|\wedge^{T} E(s)\right\|_{2}^{2}+c_{i}^{\prime}\left\|E(s)-\wedge^{T} E(s)\right\|_{\infty, V_{i}}^{2},
$$

with constants $c_{i}^{\prime}$ independent of $s$. Here \|\|$_{2}$ denotes the $L^{2}$-norm on $\mathbf{G}(\mathbb{Q}) \backslash G^{1}$ and \|\|$_{\infty, V_{i}}$ the $L^{\infty}$-norm (sup-norm) on $V_{i}$.

We shall estimate each term in the right hand side of (6.5) separately. Choosing $T$ sufficiently regular, we can now apply Lemma 4.2 of $\mathrm{A} 3$ in which one only needs to stay away from the poles of $E(s, x)$ to conclude that

$$
\begin{aligned}
\left\|\wedge^{T} E(s)\right\|_{2} & =(s+\bar{s})^{-1}\left\{(\varphi, \varphi) e^{T(s+\bar{s})}-(M(s) \varphi, M(s) \varphi) e^{-T(s+\bar{s})}\right\} \\
& +(s-\bar{s})^{-1}\left\{(\varphi, M(s) \varphi) e^{T(s-\bar{s})}-(M(s) \varphi, \varphi) e^{-T(s-\bar{s})}\right\} .
\end{aligned}
$$

(We refer to the formula for $\Omega_{0}(\bar{z}: z)$ in the middle of page 93 of [ $\mathrm{HC}$, as well as page 242 in [L3] for the original version.)

Upon a close examination of (6.6) one sees immediately that, away from the poles of $M(s)$, the singularities coming from $(s+\bar{s})^{-1}$ and $(s-\bar{s})^{-1}$ are removable. 
One needs to recall that the adjoint of $M(s)$ is $M(\bar{s})$. While the limit on the real axis $s=\bar{s}$ is harmless for our purposes, its values on the imaginary axis are quite subtle and must be treated with care. It involves the logarithmic derivative of $M(s)$. We are indebted to Erez Lapid for pointing this out to us upon reading an earlier version of this paper.

Using L'Hospital's rule in the variable $\sigma, s=\sigma+i t$, we compute the main part of the limit, i.e. the part coming from the first term in (6.6), as

$$
2(\varphi, \varphi) T-\left(M(i t)^{-1} M^{\prime}(i t) \varphi, \varphi\right) .
$$

To proceed, we need Muller's results $[\mathrm{Mu}]$.

Write $M(s)=B(s) A(s)^{-1}$ as in Theorem 4.30 of $[\mathrm{Mu}$, with $A(s)$ and $B(s)$ meromorphic linear operators, defined in Lemma 4.27 of $[\mathrm{Mu}]$. Clearly, to understand the growth of (6.7), we need to control the operators $A(s), B(s)$, their derivatives, and their inverses. For this, one needs to control $\operatorname{det} A(s), \operatorname{det} B(s), \operatorname{det} A^{\prime}(s)$, and $\operatorname{det} B^{\prime}(s)$. Observe that by estimates (5.9) of $[\mathrm{Mu}$, there exists an entire function $P(s)$ of finite order, so that all the matrix coefficients of $P(s) A(s)$ and $P(s) B(s)$ are entire and of finite order. By Leibnitz's rule, $P(s)^{2} A^{\prime}(s)$ and $P(s)^{2} B^{\prime}(s)$ have matrix coefficients which are all entire and of finite order. From this, it is clear (cf. proof of Theorem 5.10 of $[\mathrm{Mu}]$ ) that there exists an entire function $R(s)$ of finite order such that $R(s) \operatorname{det} D(s)$ is entire and of finite order, where $D(s)$ is either $A(s), B(s), A^{\prime}(s)$, or $B^{\prime}(S)$. The fact that matrix coefficients of $M(s)$, its derivatives, and inverses are of finite order now follows from the estimate (5.9) of $\mathrm{Mu}$.

Similar statements are true about higher derivatives if needed. We recall that derivatives of an entire function of finite order are again of finite order.

It must now be repeated again that while (6.6) is valid away from the unitary axis, we must use (6.7) while we are on the axis and therefore we must understand how (6.7) behaves as $|\sigma|$ increases, i.e. as we move away from the unitary axis. By the discussion in the previous paragraph, we first observe that one can multiply (6.7) by a function (in variable $i t$ ) of exponential growth in a power of $|t|$ so that the product has the same type of growth. The same statement is true if we replace it with $\sigma+i t$ and $|t|$ with $|s|$, and in fact we can choose the multiplier to be an entire function of finite order. A similar kind of statement about (6.6) is valid on all of $\mathbb{C}$. To be precise, one will need two entire functions $\tilde{R}_{1}(s)$ and $\tilde{R}_{2}(s)$ such that the product of $\tilde{R}_{1}(s) \tilde{R}_{2}(\bar{s})$ with (6.6) is of the exponential type growth discussed above.

We can now estimate the growth of $\left\|\wedge^{2} E(s)\right\|_{2}$. In view of the preceding discussion, one sees that there exists a pair of entire functions of finite order $R_{1}(s)$ and $R_{2}(s)$ such that

$$
\left|R_{1}(s) R_{2}(\bar{s})\right|\left\|\wedge^{T} E(s)\right\|_{2}
$$

is of exponential growth in some power of $|s|$ on all of $\mathbb{C}$.

We may replace $\left|R_{2}(\bar{s})\right|$ by $\left|R_{3}(s)\right|$ in (6.8), where $R_{3}$ is another entire function of finite order. Let $Q(s)=R_{1}(s) R_{3}(s)$. We have proved:

Lemma 6.1. There exists an entire function $Q(s)$ of finite order such that

$$
|Q(s)|\left\|\wedge^{T} E(s)\right\|_{2}
$$

is of exponential growth in some power of $|s|$ on all of $\mathbb{C}$. 
Next we need to analyze

$$
\sum_{\mathbf{P}_{1}} \sum_{\delta \in \mathbf{P}_{1}(\mathbb{Q}) \backslash \mathbf{G}(\mathbb{Q})}\left|E_{P_{1}}(s, \delta u)\right| \hat{\tau}_{\mathbf{P}_{1}}\left(H_{P_{1}}(\delta u)-T\right)
$$

if $s \in T_{\varepsilon, I}$ for some $\varepsilon$ and $I$. For this we apply Corollary 5.2 of [A2] to each $E_{P_{1}}$. We disregard $y$ in the corollary or, said in other words, fix it and absorb it in $T$. Fix $N \geq 0$. Then by the corollary, (6.9) is bounded by

$$
c\|u\|^{N^{\prime}} \sup _{x \in G^{1}}\left(\left|E_{P_{1}}(s, x)\right| \cdot\|x\|^{-N}\right),
$$

where $c$ and $N^{\prime}$ are as in the corollary. We shall now use the well-known explicit expression for $E_{P_{1}}(x)$. It is

$$
E_{P_{1}}(s, x)=\sum_{w \in \Omega\left(\mathfrak{a}, \mathfrak{a}_{1}\right)}(M(s \tilde{\alpha}, w) \varphi)(x) \exp \left\langle w(s \tilde{\alpha})+\rho_{\mathbf{P}_{1}}, H_{P_{1}}(x)\right\rangle,
$$

with the obvious meaning for the Weyl set $\Omega\left(\mathfrak{a}, \mathfrak{a}_{1}\right)$. We need to estimate

$$
\sum_{\Omega\left(\mathfrak{a}, \mathfrak{a}_{1}\right)}|(M(s \tilde{\alpha}, w) \varphi)(x)|\left|\exp \left\langle w(s \tilde{\alpha})+\rho_{\mathbf{P}_{1}}, H_{P_{1}}(x)\right\rangle\right|
$$

if $s \in T_{\varepsilon, I}$, i.e. under the assumption that $|\operatorname{Re}(s)|$ is bounded and $\operatorname{Im}(s) \neq 0$, for each $\mathbf{P}_{1}$.

We start by recalling some well-known facts. The function $\varphi$ on which our Eisenstein series is built, being both $K_{M}$-finite, $K_{M}=K \cap M=K \cap M^{1}$, and $\mathfrak{z}_{M}$-finite,

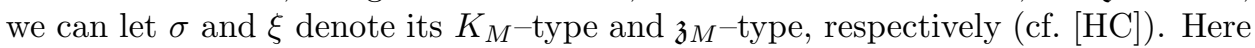
$\mathfrak{z} M$ is the center of the universal enveloping algebra of the complexified Lie algebra of (the connected component of 1 in) $M_{\mathbb{R}}^{1}$, where $P_{\infty}=\mathbf{P}(\mathbb{R})=M_{\mathbb{R}}^{1} \mathbf{A}(\mathbb{R})^{0} \mathbf{N}(\mathbb{R})$ is the Langlands decomposition of $P_{\infty}=\mathbf{P}(\mathbb{R})$. Denote by ${ }^{0} L^{2}\left(\mathbf{M}(\mathbb{Q}) \backslash M^{1}, \sigma, \xi\right)$ the space of (smooth) cusp forms on $M^{1}$ which transform according to $\sigma$ and $\xi$. Then ${ }^{0} L^{2}\left(\mathbf{M}(\mathbb{Q}) \backslash M^{1}, \sigma, \xi\right)$ is finite dimensional and of course $\varphi$ belongs to it. We need the following well-known estimate which is an immediate consequence of finiteness of dimension and boundedness of cusp forms.

There exists a constant $c>0$ such that

$$
\|\varphi\|_{\infty}=\sup _{x \in M^{1}}|\varphi(x)| \leq c\|\varphi\|_{2}
$$

for all $\varphi$ in ${ }^{0} L^{2}\left(\mathbf{M}(\mathbb{Q}) \backslash M^{1}, \sigma, \xi\right)$.

(Without considering the finiteness of dimension we can argue as follows. By the finiteness of volume, $\|\varphi\|_{2} \leq d\|\varphi\|_{\infty}$; this implies the continuity of the identity map from ${ }^{0} L^{2}\left(\mathbf{M}(\mathbb{Q}) \backslash M^{1}, \sigma, \xi\right)$ into itself equipped with \|\|$_{\infty}$-norm, cusp forms being bounded, and \|\|$_{2}-$ norm, respectively. The closed graph theorem then implies that this map is in fact a homeomorphism.)

Using (6.13) we then have

$$
\begin{aligned}
|(M(s \tilde{\alpha}, w) \varphi)(x)| & \leq c_{w}\|M(s \tilde{\alpha}, w) \varphi\|_{2} \\
& \leq c_{w}|M(s \tilde{\alpha}, w)|\|\varphi\|,
\end{aligned}
$$

where $\|\varphi\|=\|\varphi\|_{2}$ and $c_{w}$ denotes the constant of equality (6.13) applied to ${ }^{0} L^{2}\left(\mathbf{M}(\mathbb{Q}) \backslash M^{1}, \sigma^{w}, \xi^{w}\right)$ with obvious meanings for $\sigma^{w}$ and $\xi^{w}$. Observe that $c_{w}$ only depends on this space and not $s$. If we now again apply Lemma 101 of [HC] (or the discussion on page 242 of [L3]), we see that (6.12) is bounded by a constant multiple of $\|\varphi\|$ in every $T_{\varepsilon, I}$ with $I \geq 0$. 
Since $s \in T_{\varepsilon, I}$, which in particular implies that $|\operatorname{Re}(s)|$ is bounded, part b) of Lemma 5 of [HC] shows that there exist a constant $d_{w}>0$ and an integer $M_{w}$ such that

$$
\left|\exp \left\langle w(s \tilde{\alpha})+\rho_{\mathbf{P}_{1}}, H_{P_{1}}(x)\right\rangle\right| \leq d_{w}\|x\|^{M_{w}} .
$$

Here $\|x\|$ denotes the norm of $x$ (cf. [르,,$[\mathrm{HC})$.

Using the estimates (6.13), (6.14), and (6.15) in (6.12), we now conclude that there exist a constant $\kappa>0$ and an integer $L \geq 0$ such that

$$
\left|E_{P_{1}}(s, x)\right| \leq \kappa\|x\|^{L}
$$

for all $s$ in the given $T_{\varepsilon, I}$ with $I \geq 0$. Now choose $N$ in (6.10) equal to $L$ to conclude:

Lemma 6.2. Given every $T_{\varepsilon, I}$ with $I \geq 0$, there exist a constant $c>0$ (depending also on $\sigma$ and $\xi$ ) and an integer $N \geq 0$ such that

$$
\sum_{\delta \in \mathbf{P}_{1}(\mathbb{Q}) \backslash \mathbf{G}(\mathbb{Q})}\left|E_{P_{1}}(s, \delta x)\right| \hat{\tau}_{\mathbf{P}_{1}}\left(H_{P_{1}}(\delta x)-T\right) \leq c\|\varphi\|\|x\|^{N}
$$

for all $x \in G^{1}$ and all $\varphi \in{ }^{0} L^{2}\left(\mathbf{M}(\mathbb{Q}) \backslash M^{1}, \sigma, \xi\right)$.

Remark 1. Observe how important the boundedness of $|\operatorname{Re}(s)|$ is.

Remark 2. The constants $c$ and $N$ need not be the same as in (6.10).

Remark 3. This is the adelic version of Lemma 91 of [HC].

Proposition 5.4 is now a consequence of Lemmas 6.1 and 6.2 applied to estimates (6.4) and (6.5).

\section{REFERENCES}

[A1] J. Arthur, Intertwining operators and residues I: Weighted characters, J. Funct. Anal. 84 (1989), 19-84. MR 90j:22018

[A2] - A trace formula for reductive groups I: Terms associated to classes in $G(\mathbb{Q})$, Duke Math. J. 45 (1978), 911-952. MR 80d:10043

[A3] A trace formula for reductive groups II: Applications of a truncated operator, Comp. Math. 40 (1980), 87-121. MR 81b:22018

[A4] Endoscopic L-functions and a combinatorial identity, Dedicated to H. S. M. Coxeter on the occasion of his 90th birthday, Canad. J. Math. 51 (1999), no. 6, 11351148. CMP 2000:12

[B] A. Borel, Automorphic L-functions, Proc. Sympos. Pure Math. 33, Vol. II, AMS (1979), 27-61. MR 81m:10056]

[BGH] D. Bump, D. Ginzburg, and J. Hoffstein, The symmetric cube, Invent. Math. 125 (1996), 413-449. MR 97j:11023

[CSh] W. Casselman and F. Shahidi, On irreducibility of standard modules for generic representations, Ann. Scient. Éc. Norm. Sup. 31 (1998), 561-589. MR 99f:22028

[CPS1] J.W. Cogdell and I.I. Piatetski-Shapiro, Converse theorems for $G L_{n}$, Publ. Math. I.H.E.S. 79 (1994), 157-214. MR 95m:22009

[CPS2] Unitarity and functoriality, Geo. Funct. Ann. 5 (1995), 164-173. MR 97b:22024

[CPS3] , Converse Theorems for $G L_{n}$, II, J. reine. angew. Math. 507 (1999), 165-188. MR 2000a:22029

[CPS4] Converse theorems for $G L_{n}$ and their application to liftings, Proceedings of International Conference on Cohomology of Arithmetic Groups, $L$-functions, and Automorphic Forms, Tata Institute of Fundamental Research, December 98-January 99 (to appear).

[CKPSS] J.W. Cogdell, H. Kim, I.I. Piatetski-Shapiro, and F. Shahidi, On lifting from classical groups to $G L_{N}$, Publ. Math. I.H.E.S. (to appear). 
[Ga] P. Garrett, Decomposition of Eisenstein series: Rankin triple product, Annals of Math. 125 (1987), 209-237. MR 88m:11033

[GJ] S. Gelbart and H. Jacquet, A relation between automorphic representations of $G L(2)$ and GL(3), Ann. Scient. Éc. Norm. Sup. 11 (1978), 471-552. MR 81e:10025

[GPSR] S. Gelbart, I. Piatetski-Shapiro, and S. Rallis, Explicit construction of automorphic L-functions, Lecture Notes in Math. 1254 (1987). MR 89k:11038

[GRS1] D. Ginzburg, S. Rallis and D. Soudry, Self dual automorphic GL $L_{n}$ modules and construction of a backward lifting from $G L_{n}$ to classical groups, IMRN 14 (1997), 687-701. MR 98e:22013

[GRS2] A local correspondence between supercuspidal representations of $G L_{2 n}(F)$ and $\tilde{S} p_{2 n}(F)$, preprint.

[GW] R. Goodman and N.R. Wallach, Whittaker vectors and conical vectors, J. Funct. Anal. 39 (1980), 199-279. MR 82i:22018

[HC] Harish-Chandra, Automorphic forms on semisimple Lie groups, SLN, Vol. 62, BerlinHeidelberg-New York, 1968. MR 38:1216

[HT] M. Harris and R. Taylor, On the geometry and cohomology of some simple Shimura varieties, preprint.

[H] G. Henniart, Une preuve simple des conjecture de Langlands pour $G L(n)$ sur un corps p-adique, Invent. Math. 139 (2000), 439-455. CMP 2000:07

[I] T. Ikeda, On the location of poles of the triple L-functions, Comp. Math. 83 (1992), 187-237. MR 94b:11042

[J] H. Jacquet, Fonctions de Whittaker associeés aux groups de Chevalley, Bull. Soc. Math. France 95 (1967), 243-309. MR 42:6158

[JS1] H. Jacquet and J.A. Shalika, On Euler products and the classification of automorphic representations I, Amer. J. Math. 103 (1981), 499-558. MR 82m:10050a

[JS2] - On Euler products and the classification of automorphic representations II, Amer. J. Math. 103 (1981), 777-815. MR 82m:10050b

[JPSS] H. Jacquet, I.I. Piatetski-Shapiro, and J.A. Shalika, Rankin-Selberg convolutions, Amer. J. Math. 105 (1983), 367-464. |MR 85g:11044

[K1] H. Kim, Langlands-Shahidi method and poles of automorphic L-functions: Applications to exterior square L-functions, Canad. J. Math. 51 (1999), 835-849. MR 2000f:11058

[K2] Langlands-Shahidi method and poles of automorphic L-functions II, Israel J. Math. 117 (2000), 261-284. CMP 2000:13

[K3] Lifting from $G L_{4}$ to $G L_{6}$; Exterior square lift, preprint.

[KSh1] H. Kim and F. Shahidi, Symmetric cube L-functions for $G L_{2}$ are entire, Annals of Math. 150 (1999), 645-662. CMP 2000:05

[KSh2] - Holomorphy of Rankin triple L-functions; special values and root numbers for symmetric cube L-functions, Israel J. Math. (to appear).

[KSh3] On the holomorphy of certain L-functions, in preparation.

[KSh4] Functorial products for $G L_{2} \times G L_{3}$ and functorial symmetric cube for $G L_{2}$, Comptes Rendus de L'Académie des Sciences (to appear).

[Ku] P. Kutzko, The Langlands conjecture for $G L_{2}$ of a local field, Annals of Math. 112 (1980), 381-412. MR 82e:12019

[L1] R.P. Langlands, Euler Products, Yale University Press, New Haven, 1971. MR 54:7387

[L2] _ On the functional equations satisfied by Eisenstein series, Lecture Notes in Math. SLN 544, Berlin-Heidelberg-New York, 1976. MR 58:28319

[L3] Eisenstein series, in Algebraic Groups and Discontinuous Subgroups, Proc. Sympos. Pure Math. Vol. 9, AMS, 1965, pp. 235-257. MR 40:2784

[L4] On the classification of irreducible representations of real algebraic groups, in Representation Theory and Harmonic Analysis on Semisimple Lie groups (P.J. Sally, Jr. and D.A. Vogan, eds.), Mathematical Surveys and Monographs, vol. 31, AMS, 1989, pp. 101-170. MR 91e:22017

[Le] B. Ya. Levin, Lectures on Entire Functions; In Collaboration with Yu. Lyubarskii, M. Sodin, and V. Tkachenko, Trans. Math. Mono. 150; AMS, 1996. MR 97j:30001

[MW1] C. Moeglin and J.-L. Waldspurger, Spectral decomposition and Eisenstein series, Cambridge Tracts in Math., vol. 113, Cambridge University Press, 1995. MR 97d:11083

[MW2] Le spectre résiduel de $G L(n)$, Ann. Scient. Éc. Norm. Sup. 22 (1989), 605-674. MR 91b:22028 
[M1] G. Muić, Some results on square integrable representations; irreducibility of standard representations, IMRN International Math. Research Notices 14 (1998), 705-726. MR 99f:22031

[M2] , A proof of Casselman-Shahidi's conjecture for quasi-split classical groups, preprint.

[MSh] G. Muić and F. Shahidi, Irreducibility of standard representations for Iwahori-spherical representations, Math. Ann. 312 (1998), 151-165. MR 99g:22012

$[\mathrm{Mu}] \quad$ W. Müller, The trace class conjecture in the theory of automorphic forms, Ann. of Math 130 (1989), 473-529. MR 90m:11083

[Rag1] R. Raghunathan, A comparison of zeros of L-functions, Math. Res. Lett. 6 (1999), 155-167. MR 2000e: 11109

[Rag2] - Converse theorems for Dirichlet series with poles, C.R. Acad. Sci. Paris 327 (1998), 231-235. MR 99i:11034

[Ra] D. Ramakrishnan, Modularity of the Rankin-Selberg L-series, and multiplicity one for $S L(2)$, Ann. of Math. (to appear).

[RS] Z. Rudnick and P. Sarnak, Zeros of principal L-functions and random matrix theory, a celebration of John F. Nash, Jr., Duke Math. J. 81 (1996), 269-322. MR 97f:11074

[Sch] G. Schiffmann, Inteǵrales d'enterlacement et fonctions de Whittaker, Bull. Soc. Math. France 99 (1971), 3-72. MR 47:400

[Sh1] F. Shahidi, A proof of Langlands' conjecture on Plancherel measures; Complementary series for p-adic groups, Ann. of Math. 132 (1990), 273-330. MR 91m:11095

[Sh2] On the Ramanujan conjecture and finiteness of poles for certain L-functions, Ann. of Math. 127 (1988), 547-584. MR 89h:11021

[Sh3] Local coefficients as Artin factors for real groups, Duke Math. J. 52 (1985), 973-1007. MR 87m:11049

[Sh4] - On certain L-functions, Amer. J. Math. 103 (1981), 297-356. MR 82i:10030

[Sh5] - Automorphic L-functions: A Survey, in Automorphic Forms, Shimura Varieties, and $L$-functions I (L. Clozel and J.S. Milne, eds.), Perspectives in Math., vol. 10, 1990, pp. 415-437. MR 91i:11170

[Sh6] _ Fourier transforms of intertwining operators and Plancherel measures for GL(n), Amer. J. Math. 106 (1984), 67-111. MR 86b:22031

[Sh7] $\quad$ Third symmetric power L-functions for GL(2), Comp. Math. 70 (1989), 245273. MR 90m:11081

[Sh8] Symmetric power L-functions for $G L(2)$, Elliptic Curves and Related Topic (H. Kisilevsky and R. Murty, eds.), CRM Proceedings and Lecture Notes, 1994, pp. 159182. MR 95c:11066

[Sh9] Whittaker models for real groups, Duke Math. J. 47 (1980), 99-125. MR 81d:22020

[Si] A. Silberger, Special representations of reductive p-adic groups are not integrable, Ann. of Math. 111 (1980), 571-587. MR 82k:22015

[So1] D. Soudry, Rankin-Selberg convolutions for $S O_{2 \ell+1} \times G L_{n}$ : Local theory, Mem. of AMS 500, Vol. 105 (1993). MR 94b:11043

[So2] - On the archimedean theory of Rankin-Selberg Convolutions for $S O_{2 \ell+1} \times G L_{n}$, Ann. Scient. Éc. Norm. Sup. 28 (1995), 161-224. MR 96m:11043

[T] E.C. Titchmarsh, Theory of functions, Oxford Science Publications, 2nd Edition, 1991.

[Z] S. Zampera, The residual spectrum of $G_{2}$, J. Math. Pures et Appl. 76 (1997), 805-835. MR 98k:22077

[Zh] Y. Zhang, The holomorphy and nonvanishing of normalized local intertwining operators, Pacific J. Math. 180 (1997), 385-398. [MR 98k:22076]

Nicki and J. Ira Harris Professorial Chair, Weizmann Institute of Science, Rehovot 76100, ISRAEL

Department of Mathematics, Purdue University, West Lafayette, Indiana 47907

E-mail address: shahidi@math.purdue.edu 